TOBIAS ACHTERBERG

THORSTEN KOCH

AleXANDER MARTiN]

\title{
Branching Rules revisited
}




\title{
Branching Rules Revisited
}

\author{
Tobias Achterberg ${ }^{\dagger} \quad$ Thorsten Koch $^{\ddagger} \quad$ Alexander Martin ${ }^{\S}$
}

May 5, 2004

\begin{abstract}
Mixed integer programs are commonly solved with linear programming based branch-and-bound algorithms. The success of the algorithm strongly depends on the strategy used to select the variable to branch on.

We present a new generalization called reliability branching of today's state-of-the-art strong branching and pseudocost branching branching strategies for linear programming based branch-and-bound algorithms. After reviewing commonly used branching strategies and performing extensive computational studies we compare different parameter settings and show the superiority of our proposed new strategy.
\end{abstract}

Keywords: Mixed-integer-programming, Branch-and-Bound, Variable selection, Pseudocost-Branching, Strong-Branching, Reliability-Branching

\section{Introduction}

In this paper we are dealing with mixed integer programs (MIPs), which are optimization problems of the following form:

$$
c^{\star}=\min c^{T} x, \quad A x \leq b, \quad x \in \mathbb{Z}^{I} \times \mathbb{R}^{N \backslash I},
$$

where $A \in \mathbb{R}^{m \times n}, b \in \mathbb{R}^{m}, c \in \mathbb{R}^{n}$ and $I \subseteq N=\{1, \ldots, n\}$.

Among the most successful methods are currently linear programming based branch-and-bound (B\&B) algorithms where the underlying linear programs (LPs) are possibly strengthened by cutting planes. Most commercial integer programming solvers, see [7, are based on this method. As we will see below, B\&B algorithms leave two choices: how to split a problem (branching) and which (sub)problem to select next. In this paper we focus on the branching step and introduce a new generalization that contains

\footnotetext{
${ }^{\dagger}$ Konrad-Zuse-Zentrum für Informationstechnik Berlin, achterberg@zib.de

${ }^{\ddagger}$ Konrad-Zuse-Zentrum für Informationstechnik Berlin, koch@zib.de

${ }^{\S}$ Technische Universität Darmstadt, Schloßgartenstr. 7, D-64289 Darmstadt, Germany, martin@mathematik.tu-darmstadt.de
} 
many of the known branching rules as special cases. We show that specific choices of parameters for this new class of branching rules perform in most cases better than the current rules when tested on real-world instances.

In Section 2 we review current branching strategies from the literature and present our new generalization. In Section 3 the results of extensive numerical tests on specific parameter choices are presented.

We use the following notation. $X_{\text {MIP }}$ denotes the set of feasible solutions of (11), and we set $c^{\star}=\infty$ if $X_{\mathrm{MIP}}=\emptyset$. The linear programming relaxation of (1I) is obtained by removing the integrality constraints:

$$
\bar{c}_{P_{\mathrm{LP}}}=\min \left\{c^{T} x \mid x \in P_{\mathrm{LP}}\right\},
$$

where $P_{\mathrm{LP}}=\left\{x \in \mathbb{R}^{n} \mid A x \leq b\right\}$. We also denote $\bar{c}_{P_{\mathrm{LP}}}=\infty$ if $P_{\mathrm{LP}}=\emptyset$. Obviously, $\bar{c}_{P_{\mathrm{LP}}} \leq c^{\star}$, since $P_{\mathrm{LP}} \supseteq X_{\mathrm{MIP}}$. A typical LP based B\&B algorithm for solving (11) looks as follows:

Algorithm 1 (LP based branch-and-bound)

Input: A MIP in the form (11).

Output: An optimal solution $x^{\star} \in X_{\mathrm{MIP}}$ and its value $c^{\star}=c^{T} x^{\star}$ or the conclusion that $X_{\mathrm{MIP}}=\emptyset$, denoted by $c^{\star}:=\infty$.

1. Initialize the problem set $S:=\left\{P_{\mathrm{LP}}\right\}$ with the LP relaxation of the MIP. Set $c^{\star}:=\infty$.

2. If $S=\emptyset$, exit by returning the value $c^{\star}$ (with an optimal solution $x^{\star}$ ).

3. Choose a problem $Q \in S$ and delete it from $S$.

4. Solve the linear program $\bar{c}_{Q}=\min \left\{c^{T} x \mid x \in Q\right\}$ with optimal solution $\bar{x}_{Q}$, where $Q$ might have been strengthened by cutting planes.

5. If $\bar{c}_{Q} \geq c^{\star}$, goto 2 ,

6. If $\bar{x}_{Q} \in X_{\mathrm{MIP}}$, set $c^{\star}:=\bar{c}_{Q}$ and $x^{\star}:=\bar{x}_{Q}$, and goto 2 ,

7. Branching: Split $Q$ into subproblems, add them to $S$ and goto 3

If it is clear from the context we omit $Q$ from all parameters and write $\bar{c}, \bar{x}$, etc. instead of $\bar{c}_{Q}, \bar{x}_{Q}$, etc.

\section{$2 \quad$ Branching Rules}

Since branching is in the core of any B\&B algorithm, finding good strategies was important to practical MIP solving right from the beginning [3, 13]. We refrain from giving details of all existing strategies, but concentrate on the most popular rules used in todays MIP solvers. For a comprehensive study of $\mathrm{B} \& \mathrm{~B}$ strategies we refer to [8, 10] and the references therein.

The only way to split a problem $Q$ within an LP based B\&B algorithm is to branch on linear inequalities in order to keep the property of having an LP 
relaxation at hand. The easiest and most common inequalities are trivial inequalities, i.e., inequalities that split the feasible interval of a singleton variable. To be more precise, if $i$ is some variable with a fractional value $\bar{x}_{i}$ in the current optimal LP solution, we set $f_{i}^{+}=\left\lceil\bar{x}_{i}\right\rceil-\bar{x}_{i}$ and $f_{i}^{-}=\bar{x}_{i}-\left\lfloor\bar{x}_{i}\right\rfloor$. We obtain two subproblems, one by adding the trivial inequality $x_{i} \leq\left\lfloor\bar{x}_{i}\right\rfloor$ (called the left subproblem or left son, denoted by $Q_{i}^{-}$) and one by adding the trivial inequality $x_{i} \geq\left\lceil\bar{x}_{i}\right\rceil$ (called the right subproblem or right son, denoted by $Q_{i}^{+}$). This rule of branching on trivial inequalities is also called branching on variables, because it only requires to change the bounds of variable $i$. Branching on more complicated inequalities or even splitting the problem into more than two subproblems are rarely incorporated into general MIP solvers, even though it can be effective in special cases, see, for instance, [4, [5], or [15].

The basic algorithm for variable selection may be stated as follows:

Algorithm 2 (generic variable selection)

Input: Current subproblem $Q$ with an optimal LP solution $\bar{x} \notin X_{\mathrm{MIP}}$.

Output: An index $i \in I$ of a fractional variable $\bar{x}_{i} \notin \mathbb{Z}$.

1. Let $C=\left\{i \in I \mid \bar{x}_{i} \notin \mathbb{Z}\right\}$ be the set of branching candidates.

2. For all candidates $i \in C$, calculate a score value $s_{i} \in \mathbb{R}$.

3. Return an index $i \in C$ with $s_{i}=\max _{j \in C}\left\{s_{j}\right\}$.

In the following we focus on the most common variable selection rules, which are all variants of Algorithm 2. The difference is how the score in Step 2 is computed.

The ultimate goal is to find a fast branching strategy that minimizes the number of B\&B nodes that need to be evaluated. Since a global approach is unlikely, one tries to find a branching variable that is at least a good choice for the current branching. The quality of a branching is measured by the change in the objective function of the LP relaxations of the two children $Q_{i}^{-}$and $Q_{i}^{+}$compared to the relaxation of the parent node $Q$.

In order to compare branching candidates, for each candidate the two objective function changes $\Delta_{i}^{-}:=\bar{c}_{Q_{i}^{-}}-\bar{c}_{Q}$ and $\Delta_{i}^{+}:=\bar{c}_{Q_{i}^{+}}-\bar{c}_{Q}$ are mapped on a single score value. This is typically done by using a function of the form (cf. [10])

$$
\operatorname{score}\left(q^{-}, q^{+}\right)=(1-\mu) \cdot \min \left\{q^{-}, q^{+}\right\}+\mu \cdot \max \left\{q^{-}, q^{+}\right\} .
$$

The score factor $\mu$ is some number between 0 and 1 . It is usually an empirically determined constant, which is sometimes adjusted dynamically through the course of the algorithm (in SIP we use $\mu=1 / 6$ ). Note that special treatment is necessary, if one of the subproblems $Q_{i}^{-}$or $Q_{i}^{+}$is infeasible.

In the forthcoming explanations all cases are symmetric for the left and right subproblem. Therefore we will only consider one direction, the other will be analogous. 


\subsection{Most Infeasible Branching}

This still very common rule chooses the variable with fractional part closest to 0.5 , i. e., $s_{i}=0.5-\left|\bar{x}_{i}-\left\lfloor\bar{x}_{i}\right\rfloor-0.5\right|$. The heuristic reason behind this choice is that this selects a variable where the least tendency can be recognized to which "side" (up or down) the variable should be rounded. Unfortunately, as the numerical results in Section 3 indicate, the performance of this rule is in general not better than selecting the variable randomly.

\subsection{Pseudocost Branching}

This is a sophisticated rule in the sense that it keeps a history of the success of the variables on which already has been branched. This rule goes back to [3]. In the meantime various variations of the original rule have been proposed. In the following we present the one used in SIP [12]. For alternatives see [10].

Let $\varsigma_{i}^{+}$be the objective gain per unit change in variable $i$ at node $Q$, that is

$$
\varsigma_{i}^{+}=\Delta_{i}^{+} / f_{i}^{+} .
$$

Let $\sigma_{i}^{+}$denote the sum of $\varsigma_{i}^{+}$over all problems $Q$, where $i$ has been selected as branching variable and $Q_{i}^{+}$has already been solved and was feasible. Let $\eta_{i}^{+}$be the number of these problems. Then the pseudocosts for the upward branching of variable $i$ are

$$
\Psi_{i}^{+}=\sigma_{i}^{+} / \eta_{i}^{+} .
$$

Using $s_{i}=\operatorname{score}\left(f_{i}^{-} \Psi_{i}^{-}, f_{i}^{+} \Psi_{i}^{+}\right)$in Algorithm 2 yields what is called pseudocost branching.

Observe that at the beginning of the algorithm $\sigma_{i}^{+}=\eta_{i}^{+}=0$ for all $i \in I$. We call the pseudocosts of a variable $i \in I$ uninitialized for the upward direction, if $\eta_{i}^{+}=0$. Uninitialized upward pseudocosts are set to $\Psi_{i}^{+}=\Psi_{\text {avg }}^{+}$, where $\Psi_{\text {avg }}^{+}$is the average of the initialized upward pseudocosts over all variables. This average number is set to 1 in the case that all upward pseudocosts are uninitialized. The pseudocosts of a variable are called uninitialized if they are uninitialized in at least one direction.

\subsection{Strong Branching}

The idea of strong branching, introduced in CPLEX 7.5 (see also [2]), is to test which of the fractional candidates gives the best progress before actually branching on any of them. This test is done by temporarily introducing an lower bound $\left\lfloor\bar{x}_{i}\right\rfloor$ and subsequently a upper bound $\left\lceil\bar{x}_{i}\right\rceil$ for variable $i$ with fractional LP value $\bar{x}_{i}$, and solving the linear relaxations.

If we choose as candidate set the full set $C=\left\{i \in I \mid \bar{x}_{i} \notin \mathbb{Z}\right\}$ and if we solve the resulting LPs to optimality, we call the strategy full strong branching. In other words, full strong branching can be viewed as finding 
the locally (with respect to the given score function) best variable to branch on. We will see in Section 3 that selecting this locally best variable usually works very well in practice w.r.t. the number of nodes needed to solve the problem instances.

Unfortunately the computation times per node of full strong branching are high. Accordingly most branching rules presented in literature may be interpreted as an attempt to find a (fast) estimate of what full strong branching actually measures.

One possibility to speedup full strong branching, is to restrict the candidate set in some way, e.g. by considering only a subset $C^{\prime} \subseteq C$ of the fractional variables. To estimate the changes in the objective function for a specific branching decision, often only a few simplex iterations are performed, because the change of the objective function in the simplex algorithm usually decreases with the iterations. Thus, the parameters of strong branching to be specified are the size of the candidate set $C^{\prime}$, the maximum number $\gamma$ of dual simplex iterations to be performed on each candidate variable, and a criterion according to which the candidate set is selected.

In SIP, the size of the candidate set is not fixed in advance to a specific value, but the candidates are evaluated with a "look ahead" strategy: if no new best candidate was found for $\lambda$ successive candidates, the evaluation process is stopped. By evaluating variables with largest pseudocost scores first, only the most promising candidates are evaluated. The iteration limit for strong branching evaluations is set to $\gamma=2 \bar{\gamma}$, where $\bar{\gamma}$ is the average number of simplex iterations per LP needed so far. Note that this number only protects from unexpected long simplex runs, on average the candidate LPs will be solved to optimality.

\subsection{Hybrid Strong/Pseudocost Branching}

Even with the speedups indicated at the end of Section 2.3, the computational burden of strong branching is high, and the higher the speedup, the less precise the decisions are.

On the other hand, the weakness of pseudocost branching is that at the very beginning there is no information available, and $s_{i}$ is almost identical for all variables $i \in C$. Many of the early nodes are located in the upper part of the search tree where the decisions have the largest impact on the structure of the tree and the subproblems therein. With pseudocost branching, these decisions are taken with respect to pseudocost values that aren't useful yet.

To circumvent these drawbacks the positive aspects of pseudocost and strong branching are put together in the combination hybrid strong/pseudocost branching, where strong branching is applied in the upper part of the tree up to a given depth level $d$. For nodes with depth larger than $d$, pseudocost branching is used. This branching rule is available for example in LiNDO [11. 


\subsection{Pseudocost Branching with Strong Branching Initialization}

The decisions of pseudocost as well as the ones of hybrid strong/pseudocost branching in the lower part of the tree are potentially based on uninitialized pseudocost values, leading to an inferior selection of branching variables.

The idea to avoid this risk, which goes back to [10, is to use strong branching for variables with uninitialized pseudocosts and to use the resulting strong branching estimates to initialize the pseudocosts. In contrast to the fixed depth level of hybrid strong/pseudocost branching, this rule uses strong branching in a more dynamic way.

\subsection{Reliability Branching}

We generalize the idea of pseudocost branching with strong branching initialization by not only using strong branching on variables with uninitialized pseudocost values, but also on variables with unreliable pseudocost values. The pseudocosts of a variable $i$ are called unreliable, if $\min \left\{\eta_{i}^{-}, \eta_{i}^{+}\right\}<\eta_{\mathrm{rel}}$, with $\eta_{\text {rel }}$ being the "reliability" parameter. We call this new branching rule reliability branching.

An outline of the selection of a branching variable with reliability branching is given in the following Algorithm 3 that replaces Step 2 of Algorithm 2

\section{Algorithm 3 (Reliability branching)}

2. For all candidates $i \in C$, calculate the score $s_{i}=\operatorname{score}\left(f_{i}^{-} \Psi_{i}^{-}, f_{i}^{+} \Psi_{i}^{+}\right)$ and sort them in non-increasing order of their pseudocost scores.

For all candidates $i \in C$ with $\min \left\{\eta_{i}^{-}, \eta_{i}^{+}\right\}<\eta_{\text {rel }}$, do:

(a) Perform a number of at most $\gamma$ dual simplex iterations on each subproblem $Q_{i}^{-}$and $Q_{i}^{+}$. Let $\tilde{\Delta}_{i}^{-}$and $\tilde{\Delta}_{i}^{+}$be the resulting gains in the objective value.

(b) Update the pseudocosts $\Psi_{i}^{-}$and $\Psi_{i}^{+}$with the gains $\tilde{\Delta}_{i}^{-}$and $\tilde{\Delta}_{i}^{+}$.

(c) Update the score $s_{i}=\operatorname{score}\left(\tilde{\Delta}_{i}^{-}, \tilde{\Delta}_{i}^{+}\right)$.

(d) If the maximum score $s^{*}=\max _{j \in C}\left\{s_{j}\right\}$ has not changed for $\lambda$ consecutive score updates, goto 3 . 


\subsection{Branching Rule Classification}

Some of the proposed branching rules can be adjusted with parameter settings. All of the strategies using strong branching include the simplex iteration limit $\gamma$ and the look ahead value $\lambda$. The hybrid strong/pseudocost branching exhibits an additional depth parameter $d$, while the reliability branching comes along with the reliability parameter $\eta_{\text {rel }}$.

It is interesting to note that depending on the parameter settings, the branching rules have interrelations as illustrated in Figure 1

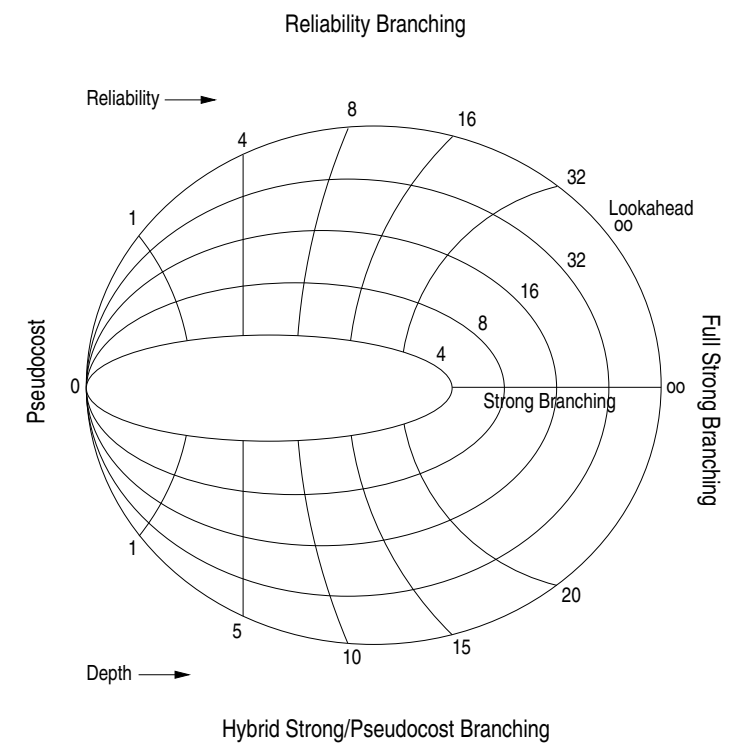

Figure 1. Interrelations between branching rules and their parameters

Hybrid strong/pseudocost branching with $d=0$ as well as reliability branching with $\eta_{\mathrm{rel}}=0$ coincide with pure pseudocost branching. With $\eta_{\mathrm{rel}}=1$, reliability branching is equal to pseudocost branching with strong branching initialization. If the depth $d$ and the reliability $\eta_{\text {rel }}$ are increased, the number of strong branching evaluations also increases, and with $d=$ $\eta_{\text {rel }}=\infty$, both strategies converge to pure strong branching. Additionally, if the look ahead parameter is set to $\lambda=\infty$, strong branching becomes full strong branching.

Reliability $\left\{\begin{array}{l}\eta_{\mathrm{rel}}=0 \\ \eta_{\mathrm{rel}}=1 \\ \eta_{\mathrm{rel}} \in[4,8] \\ \eta_{\mathrm{rel}}=\infty\end{array}\right\}$ yields $\left\{\begin{array}{l}\text { pseudocost branching } \\ \text { pseudocost branching with } \\ \text { strong branching initialization } \\ \text { best performing branching rules } \\ \text { strong branching }\end{array}\right.$ 


\section{Computational Results}

In this section we compare computational results for different branching rules and parameter settings on several MIP instances. All calculations were performed on a $833 \mathrm{MHz}$ Alpha 21264 workstation with $4 \mathrm{MB}$ Cache and 2 GB RAM.

\subsection{Test Set}

Our test set consists of instances from MiPLIB 2003 [1 and instances used by [14. We selected all problems where CPLEX 9.0 needs at least 5000 branching nodes and at most one hour CPU time for solving. (CPLEX was run with default settings, except that "absolute mipgap" was set to $10^{-10}$ and "relative mipgap" to 0.0, which are the corresponding values in SIP.)

In all runs, we used a time limit of 3600 seconds. Note that the version of SIP used here utilizes CPLEx 9.0 as embedded LP solver. The strong branchings are performed using CPXstrongbranch().

What makes benchmarking branching strategies difficult are the complex interrelations between cutting plane generation, primal heuristics, node selection, and branching variable selection. For example, it is possible that a "worse" branching rule results in less branching nodes and a smaller solution time for a specific instance, because the variable selection leads incidentally to an early discovering of a good or optimal primal solution. However, we dispensed with setting the optimal solution values beforehand, since leading towards feasible solutions fast is a desirable property of branching rules. 1]

Hence, for our comparison of the branching strategies we used the default parameter settings except that cuts are generated in the root node only. For this parameter setting, which is commonly known as cut-and-branch, the influence of the branching strategy is emphasized best.

To verify that the branch-and-bound environment we used with SIP is state-of-the-art, we also ran CPLEX 9.0 on our test set. To better compare

\footnotetext{
${ }^{1} \mathrm{~A}$ better primal solution impacts the solution process in several different ways:

- Fewer nodes have to be evaluated, due to earlier cutoff.

- Less time per node is spend, because the dual simplex algorithm can stop when it reaches the upper bound. The same applies to the strong branching evaluations.

- The average depth of nodes that use strong branching decreases, because of the node selection algorithm. The bigger the gap between upper and lower bound the more the selection algorithm tries to find feasible solutions by selecting nodes in a depthfirst fashion. If the gap becomes small it resembles more a best-first algorithm, and that causes reliability branching to resemble more a hybrid strong/pseudocost branching, which as you can see in Figure 2 is inferior.

- The behavior of the diving heuristic, that regularly "dives" down the tree to find feasible solutions is also influenced. This is important as pseudocosts are updated while diving.
} 
the branching decisions we used SIP's preprocessing and cutting plane generation also in CPLEX instead of CPLEX' own routines. In this way both, CPLEX and SIP, operated with pure branch and bound on the same problems. In CPLEX we used the default branching strategy which to the best of our knowledge is some variant of pseudocost branching.

\subsection{Description of Tables and Figures}

In Table 1, the summary of all computational results is presented. Columns labeled "total" give the sums of the results over all instances, columns labeled "geom." give the geometric mean over all instances. The last column lists how many instances where not solved to optimality because the limit of one hour CPU time was reached.

Tables 2 and 3 describe in detail some of our computational results. These tables show for each instance and each branching strategy the number of nodes explored and the time needed to solve all the instances. The number put in parentheses behind the branching rules is the reliability setting for reliability branching and the depth setting for hybrid strong/pseudocost branching (str/ps).

Numbers in bold face indicate the "winner" for a particular instance between hybrid strong/pseudocost branching with $d=10$, reliability branching with $\eta_{\text {rel }}=1$ which is equal to pseudocost branching with strong branching initialization, and reliability branching with $\eta_{\text {rel }}=8$. This is done separately for $\lambda=4$ and $\lambda=8$.

We did not not base our conclusions on performances of single instances and discuss those in detail Whe rather rely on average numbers over all instances. It is common sense that the geometric mean is a fair criterion for comparison and we used it as the basis for our conclusions in the next section.

One interesting observation in Table 3 is that most infeasible branching is basically as good as random branching showing that this rule is of no use. We refrain from considering both rules any further in the following discussion.

Table 4 gives the number of strong branching evaluations performed, i. e., the number of CPXstrongbranch () calls, which is the number of times a fractional variable was evaluated with strong branching by solving its two subproblems 3

\footnotetext{
${ }^{2}$ The detailed results can be found starting at page 20 in Tables 5 to 44

${ }^{3}$ Every strategy employing strong branching can detect infeasibilities and cutoffs already in the strong branching evaluation of a fractional variable, if one or both of the two subproblems are infeasible or have dual bounds exceeding the current primal bound. These fathomed subproblems are actually not generated and therefore not counted as nodes in the tables. Therefore, full strong branching (and to a lesser extend strong branching) saves the generation of the final leaves, thus reducing the number of node evaluations by a factor of $\frac{1}{2}$. This reduction of node counts is neglectable for all other branching strategies.
} 
The last column shows the average depth of the node tree as encountered while doing full strong branching. This gives an indication on how balanced the trees are, the smaller the number the more balanced the trees are. Examples can be seen in Figure 5 which display the trees of vpm2 with a low average depth and neos 3 with a very high average depth.

\subsection{Results}

We have performed a comprehensive computational study of all variants of branching strategies discussed in this paper. For each parameter setting, reflected by an intersection point of two lines in Figure 1, we ran all instances. The total number as well as the geometric mean in terms of B\&B nodes, time and strong branching evaluations is shown in Table 1 for all of these runs.

Figure 2]illustrates the geometric means of all runs. The circles, squares and stars indicate runs with reliability branching, hybrid strong/pseudocost branching, and strong branching, respectively. The CPLEX run is marked with an ' $x$ '. The numbers give, depending on the branching strategy, information about the parameters $\eta_{\mathrm{rel}}, d$, and $\lambda$.

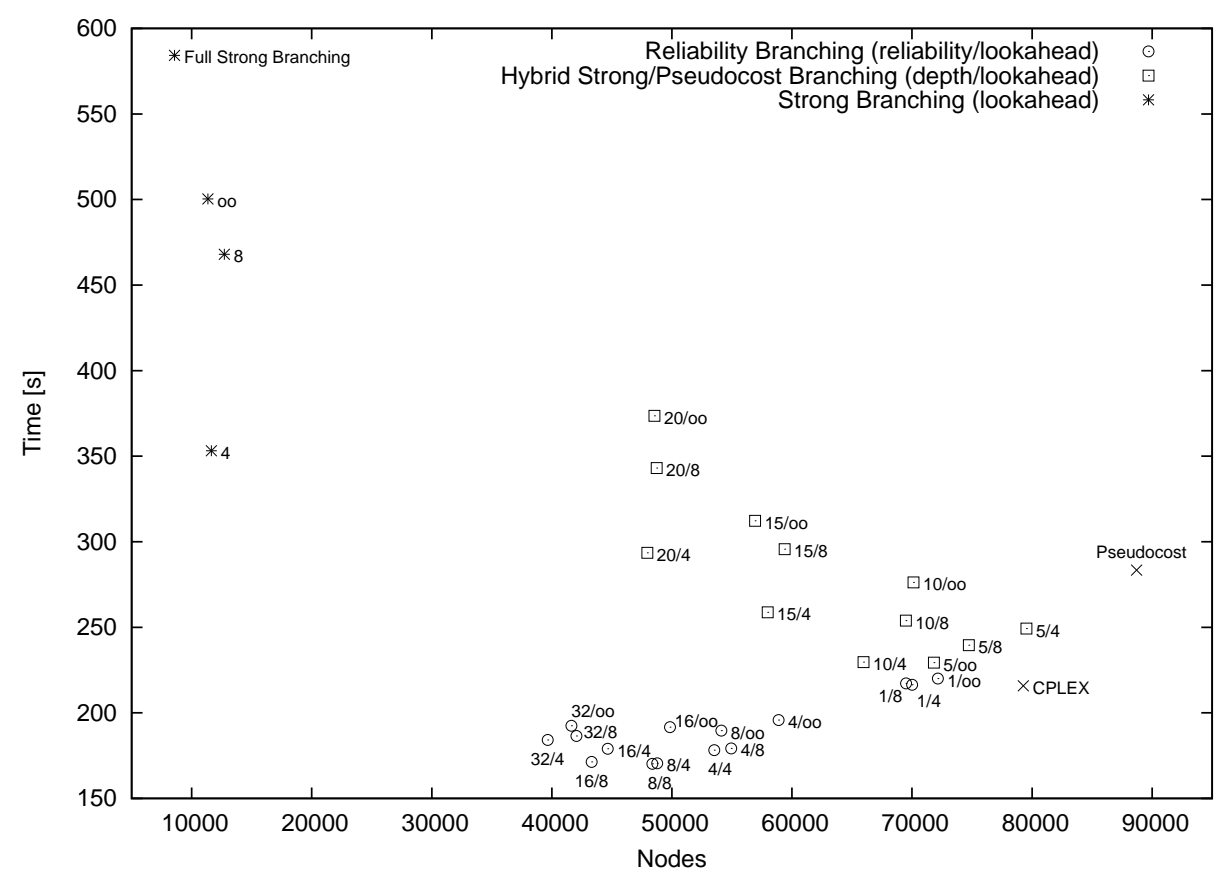

Figure 2. Nodes vs. time

The following conclusions may be drawn from these numbers:

(i) Strong branching (stars in Figure 2) performs remarkably well with respect to the number of evaluated nodes, but, as expected, not with 
respect to time. Only two instances could not be solved by full strong branching within 3600 seconds. Without time limit, qiu is solved in 15659 nodes and 11133 seconds, while neos7 is solved in 476601 nodes and 33418 seconds.

The only instance where none of the variants of strong branching needs the least number of nodes is neos7. Especially unexpected is that full strong branching needs to evaluate considerably more nodes than pseudocost branching. This is due to the fact that pseudocost branching is (incidentally) able to find the optimal solution very early (after two minutes and 15277 nodes). Note that as a consequence pseudocost branching is more than twice as fast per node than most infeasible branching.

(ii) With respect to time, regardless of the specific parameter setting, reliability branching always outperforms hybrid strong/pseudocost branching, as can be seen by comparing the circles with the rectangles in Figure 2

At least one reason why hybrid strong/pseudocost branching performs worse than reliability branching can be seen in Figure 5. What is shown are the branch-and-bound trees generated by full strong branching visualized with VBCTOOL [9]. The first tree is from vpm2 and is reasonably balanced. The second one is from neos3 and looks like a path. The right branch (fixing a variable to one) is nearly always infeasible after two variables are fixed. Since hybrid strong/pseudocost branching uses a fixed depth for deciding where to do strong branching, only very few strong branching evaluations are performed, as shown in Table 4.

The last column of Table 4 gives the average depth of all nodes generated when using full strong branching. If one compares this to the depth setting of hybrid strong/pseudocost branching and looks at the number of strong branchings performed, see also Table 4 . the difficulties with strategies based on some fixed setting become obvious.

(iii) The lookahead $\lambda$ from a certain value upwards doesn't seem to have much influence on the number of nodes.

The higher the settings the more time is spent per node evaluation, which seems not to pay off. This is not really a surprise recalling that a lookahead of four means, no new best candidate found in four consecutive tries. Since the candidates are already ordered by pseudocost value and the lookahead counter is reset with every new best candidate found, a setting of four turns out to be good enough to find the overall best candidate in most cases. 
(iv) Increasing the reliability $\eta_{\text {rel }}$ in reliability branching or the depth $d$ in hybrid strong/pseudocost branching decreases the number of evaluated nodes as expected.

See Figure 3, where the ratio of the number of nodes to strong branching evaluations is shown. With an increasing number of strong branching evaluations we are converging towards strong branching with respect to the number of evaluated nodes. The curve of reliability branching is always below the curve of hybrid strong/pseudocost branching indicating that the information provided by strong branching is used in a much better way.

Figure 4 on the other hand demonstrates the tradeoff between number of nodes and time per node. We again see that reliability branching is always better than hybrid strong/pseudocost branching. Figure 4 also nicely reflects that pseudocost branching with strong branching initialization $\left(\eta_{\mathrm{rel}}=1\right)$, see Section 2.5, is better than pseudocost branching itself $\left(\eta_{\text {rel }}=0\right)$, but this is not the best choice. The performance increases up to $\eta_{\text {rel }}=8$ and decreases with larger values again.

Looking once again at Figure 2 we see that the setting $\left(\eta_{\text {rel }}=8, \lambda=4\right)$ for reliability branching marks a new sweet spot.

\section{Conclusion}

We presented a new generalization of today's state of the art pseudocost branching and strong branching, which we call reliability branching. This strategy was implemented in SIP, an LP based branch and bound solver for mixed integer programs. The superiority of the new branching rule was demonstrated in extensive computational experiments.

It was shown that a more intensive dynamic use of strong branching leads to significant improvements in both the number of B\&B nodes and the time needed to solve the considered problem instances.

It also became evident, that there is still a gap to the number of nodes needed using full strong branching. The question is whether it is possible to bridge this gap without increasing the time spent per node too much. 


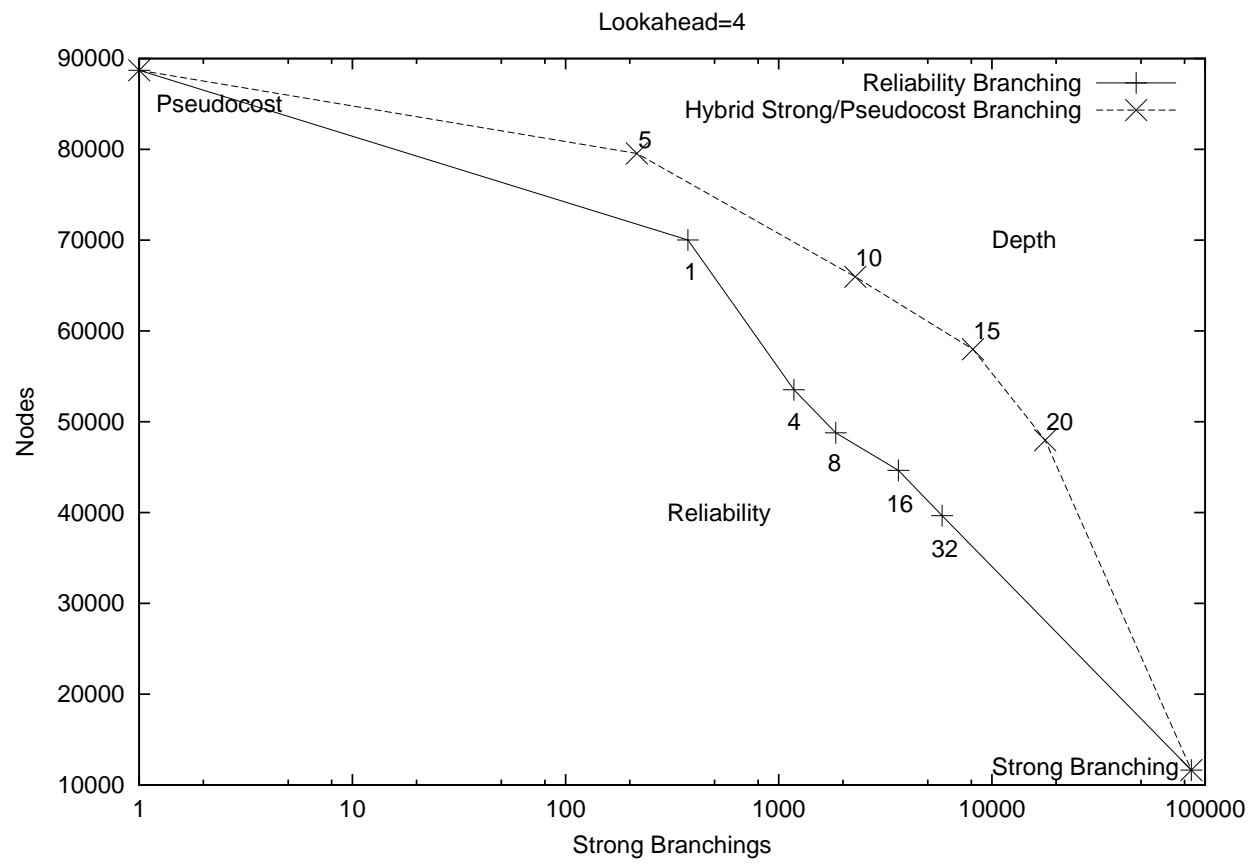

Figure 3. Nodes vs. strong branching evaluations

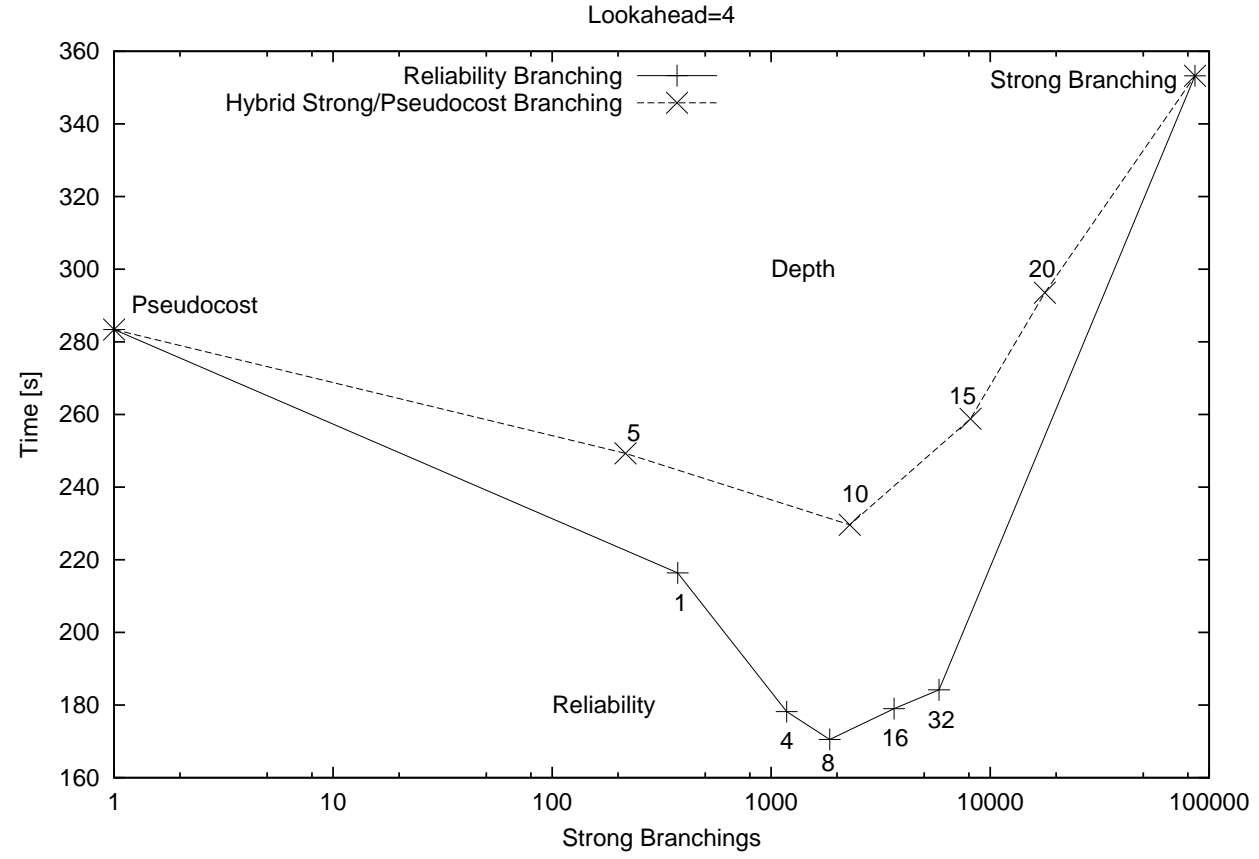

Figure 4. Time vs. strong branching evaluations 

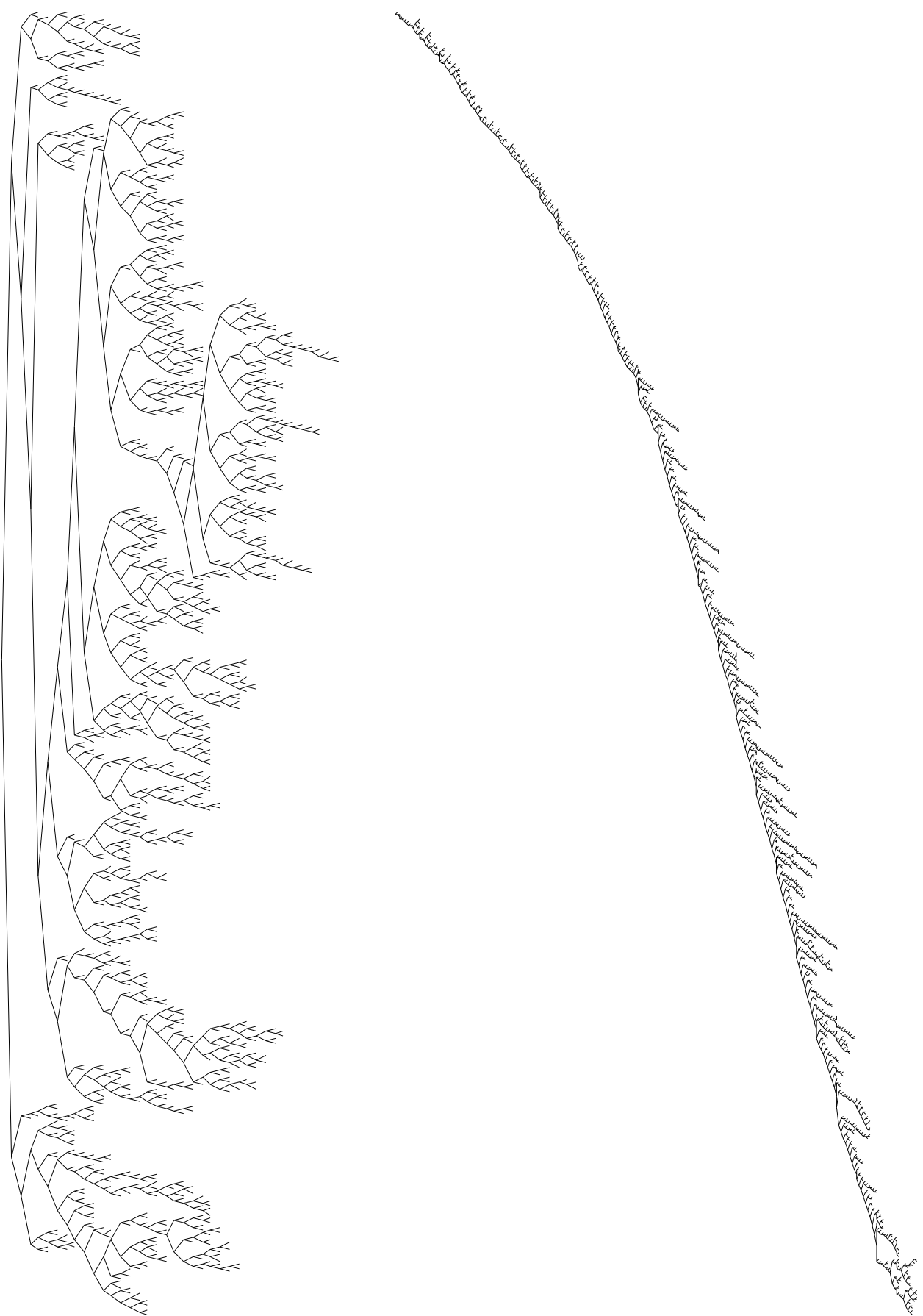

Figure 5. Comparison of node trees resulting from full strong branching for vpm2 and neos3. 


\section{References}

[1] T. Achterberg, T. Koch, and A. Martin. The mixed integer programming library: MipliB 2003, 2003. http://miplib.zib.de

[2] D. Applegate, R. E. Bixby, V. Chvátal, and W. Cook. Finding cuts in the TSP. Technical Report 95-05, DIMACS, March 1995.

[3] M. Benichou, J. M. Gauthier, P. Girodet, G. Hentges, G. Ribiere, and O. Vincent. Experiments in mixed-integer programming. Math. Prog., 1:76-94, 1971.

[4] R. Borndörfer, C.E. Ferreira, and A. Martin. Decomposing matrices into blocks. SIAM J. Optim., 9:236-269, 1998.

[5] J. M. Clochard and D. Naddef. Using path inequalities in a branch-and-cut code for the symmetric traveling salesman problem. In L. A. Wolsey and G. Rinaldi, editors, Proc. on the Third IPCO Conf., pages 291-311, 1993.

[6] ILOG CPLEX. Reference Manual, 2003. http://www.ilog.com/products/cplex.

[7] B. Fourer. 2003 Software Survey: Linear Programming. OR/MS Today, $30(6): 34-43,2003$.

[8] A. Land and S. Powell. Computer codes for problems of integer programming. Ann. of Discrete Math., 5:221-269, 1979.

[9] S. Leipert. VBCTOOL - a graphical interface for visualization of branch cut algorithms, 1996. http://www.informatik.uni-koeln.de/ls_juenger/research/vbctool

[10] J. T. Linderoth and M. W. P. Savelsbergh. A computational study of search strategies for mixed integer programming. INFORMS J. Comput., 11:173-187, 1999.

[11] LINDO. API Users Manual, 2003. http://www.lindo.com

[12] A. Martin. Integer programs with block structure. Habilitations-Schrift, Technische Universität Berlin, 1998.

[13] G. Mitra. Investigations of some branch and bound strategies for the solution of mixed integer linear programs. Math. Prog., 4:155-170, 1973.

[14] H. Mittelmann. Decision tree for optimization software: Benchmarks for optimization software, 2003. http://plato.asu.edu/bench.html

[15] D. Naddef. Polyhedral theory and branch-and-cut algorithms for the symmetric TSP. In G. Gutin and A. Punnen, editors, The Traveling Salesman Problem and its Variations. Kluwer, 2002. 
Table 1. Summary of all considered strategies

\begin{tabular}{|c|c|c|c|c|c|c|c|}
\hline \multirow{2}{*}{ Strategy } & \multicolumn{2}{|c|}{ B\&B nodes } & \multirow{2}{*}{\multicolumn{2}{|c|}{$\begin{array}{l}\text { time }(\mathrm{sec}) \\
\text { total geom. }\end{array}$}} & \multicolumn{2}{|c|}{ strong branchings } & \multirow{2}{*}{ fails } \\
\hline & total & geom. & & & total & geom. & \\
\hline random & 21246277 & 275789.4 & 46202.2 & 923.6 & 0 & 0.0 & 11 \\
\hline most infeasible & 19421589 & 262368.9 & 48037.5 & 938.0 & 0 & 0.0 & 11 \\
\hline pseudocost & 9381001 & 88706.8 & 19945.8 & 283.4 & 0 & 0.0 & 2 \\
\hline full strong & 929347 & 7241.7 & 26397.2 & 504.4 & 15569652 & 146784.2 & 2 \\
\hline \multicolumn{8}{|c|}{ lookahead = 4} \\
\hline rong/pscost (5) & 9698397 & 79535.5 & 19487.8 & 249.3 & 5792 & 216.2 & 2 \\
\hline strong/pscost (10) & 8251942 & 65966.3 & 16499.4 & 229.6 & 74812 & 2284.2 & 2 \\
\hline trong/pscost (15) & 7982847 & 57976.8 & 17855.3 & 258.8 & 523377 & 8137.8 & 2 \\
\hline strong/pscost (20) & 7890374 & 47958.5 & 19175.6 & 293.5 & 2825100 & 17780.2 & 2 \\
\hline reliability (1) & 9000334 & 70013.6 & 17199.6 & 216.4 & 39126 & 374.8 & 1 \\
\hline reliability (4) & 6906698 & 53522.9 & 13402.9 & 178.2 & 110628 & 1176.7 & 0 \\
\hline reliability (8) & 7937968 & 48772.8 & 11132.7 & 170.5 & 117643 & 1850.3 & 0 \\
\hline$(16)$ & 6022024 & 44649.9 & 10782.6 & 179.0 & 187578 & 3640.6 & 0 \\
\hline reliability (32) & 7940797 & 39655.2 & 11103.0 & 184.2 & 253014 & 5837.8 & 0 \\
\hline & 1325589 & 11639.5 & 20427.2 & 353.2 & 9965454 & 86188.6 & 3 \\
\hline \multicolumn{8}{|c|}{ lookahead $=8$} \\
\hline strong/pscost (5) & 8653318 & 74730.8 & 17389.5 & 239.6 & 7397 & 268.6 & 1 \\
\hline rong/pscost (10) & 8332543 & 69489.0 & 18362.5 & 253.9 & 102082 & 2523.8 & 2 \\
\hline rong/pscost (15) & 7456685 & 59398.8 & 20479.8 & 295.7 & 750577 & 9983.1 & 2 \\
\hline ost (20) & 7551419 & 48736.5 & 22388.8 & 343.1 & 3695577 & 20837.9 & 3 \\
\hline eliability (1) & 8663537 & 69501.0 & 16753.8 & 217.2 & 53557 & 429.0 & 1 \\
\hline eliability & 8338386 & 54937.7 & 12497.2 & 179.2 & 74906 & 1104.6 & 0 \\
\hline & 7813409 & 48377.3 & 12380.2 & 170.2 & 133545 & 1998.0 & 0 \\
\hline & 7579400 & 43311.9 & 11946.7 & 171.3 & 185136 & 3589.7 & 0 \\
\hline$y(32)$ & 7207836 & 42047.5 & 11835.7 & 186.5 & 259482 & 5913.2 & 0 \\
\hline strong branching & 1294569 & 12714.7 & 25619.4 & 468.0 & 12651504 & 119799.1 & 4 \\
\hline \multicolumn{8}{|c|}{ lookahead $=\infty$} \\
\hline strong/pscost (5) & 8498292 & 71817.4 & 18116.9 & 229.3 & 14675 & 489.9 & 2 \\
\hline strong/pscost (10) & 9247636 & 70125.8 & 20472.1 & 276.2 & 154458 & 3870.1 & 2 \\
\hline strong/pscost (15) & 6670440 & 56926.6 & 19907.4 & 312.2 & 890187 & 13127.2 & 3 \\
\hline strong/pscost (20) & 7627640 & 48547.0 & 23538.5 & 373.6 & 3842516 & 26557.4 & 3 \\
\hline reliability (1) & 7747290 & 72159.1 & 15825.8 & 220.0 & 48162 & 408.4 & 1 \\
\hline reliability (4) & 9068723 & 58886.4 & 14258.1 & 195.8 & 78625 & 1096.6 & 2 \\
\hline reliability (8) & 8551045 & 54118.3 & 13563.0 & 189.6 & 135541 & 2042.9 & 1 \\
\hline reliability (16) & 6567432 & 49839.9 & 12766.4 & 191.6 & 196220 & 3601.0 & 0 \\
\hline reliability (32) & 7502942 & 41636.1 & 12393.6 & 192.5 & 281822 & 6000.7 & 0 \\
\hline strong branching & 1163822 & 11355.7 & 26176.4 & 500.3 & 12793364 & 127737.1 & 4 \\
\hline CPLEX/SIP cuts & 10467429 & 79269.0 & 18617.4 & 215.8 & - & - & 1 \\
\hline
\end{tabular}


Table 2. B\&B nodes needed to solve each problem instance

\begin{tabular}{|c|c|c|c|c|c|c|c|c|c|c|c|c|c|}
\hline \multirow[b]{2}{*}{ Example } & \multirow[b]{2}{*}{ random } & \multirow[b]{2}{*}{ most inf. } & \multirow[b]{2}{*}{ pscost } & \multirow[b]{2}{*}{ full str. } & \multicolumn{4}{|c|}{ lookahead $=4$} & \multicolumn{4}{|c|}{ lookahead $=8$} & \multirow{2}{*}{$\begin{array}{r}\text { CPLEX } 9.0 \\
\text { (SIP cuts) }\end{array}$} \\
\hline & & & & & str/ps(10) & reli(1) & reli(8) & strong & str/ps(10) & reli(1) & reli(8) & strong & \\
\hline aflow30a & $>1248221$ & $>1025389$ & 276002 & 46649 & 164112 & 203544 & 181397 & 44455 & 210127 & 170547 & 171909 & 55478 & 105204 \\
\hline cap6000 & 7454 & 6717 & 6791 & 3402 & 4957 & 5076 & 4253 & 3452 & 4957 & 5076 & 4253 & 3452 & 4982 \\
\hline gesa2-o & $>1023765$ & $>1150438$ & 126459 & 13743 & 75141 & 57534 & 53881 & 15831 & 74673 & 79841 & 50078 & 21988 & 37293 \\
\hline mas74 & >4 662991 & $>4725208$ & 5160828 & 551780 & 5163685 & 5478830 & 5521061 & $>790014$ & 4886438 & 5296806 & 5425001 & $>645364$ & 5617512 \\
\hline mas76 & 2935689 & 2038945 & 603683 & 114228 & 587813 & 496370 & 482122 & 125516 & 666398 & 496370 & 336826 & 188882 & 507536 \\
\hline $\operatorname{misc} 07$ & 47941 & 17955 & 19407 & 2713 & 49822 & 35187 & 54932 & 5228 & 50893 & 41902 & 55740 & 5394 & 86825 \\
\hline pk1 & 1064666 & 850198 & 437758 & 56546 & 294469 & 367763 & 331339 & 128299 & 298771 & 367763 & 311611 & 143206 & 366340 \\
\hline pp08aCUTS & 1877 & 1740 & 673 & 124 & 355 & 673 & 489 & 201 & 318 & 651 & 464 & 148 & 663 \\
\hline qiu & > 163998 & $>165538$ & 15471 & $>2974$ & 25180 & 16479 & 18405 & 6178 & 23974 & 16148 & 14847 & $>6416$ & 9443 \\
\hline rout & $>714812$ & $>702392$ & 309779 & 2836 & 45457 & 44232 & 13883 & 4179 & 39212 & 39192 & 19743 & 11305 & $>1467395$ \\
\hline vpm2 & 92880 & 40258 & 22568 & 1457 & 11710 & 13780 & 9648 & 1974 & 10826 & 17409 & 10054 & 1812 & 4306 \\
\hline ran8×32 & 321933 & 674613 & 40069 & 4902 & 29957 & 26360 & 17668 & 6824 & 30526 & 31697 & 21092 & 4249 & 45143 \\
\hline 26 & $>1637058$ & $>1500171$ & 128327 & 8520 & 549 & 646 & 48065 & 279 & 48 & & 48626 & 12 & 56548 \\
\hline $\operatorname{ran} 12 \times 21$ & $>1667430$ & $>1471340$ & 234915 & 13420 & 126153 & 172161 & 135037 & 12185 & 135165 & 159383 & 124455 & 18577 & 196478 \\
\hline $\operatorname{ran} 13 \times 13$ & 907443 & 758186 & 149239 & 9147 & 86648 & 109241 & 95288 & 16033 & 86566 & 97195 & 93939 & 20516 & 97325 \\
\hline mas284 & 162255 & 123569 & 21586 & 3226 & 18700 & 24383 & 21179 & 4564 & 17799 & 21217 & 20360 & 5580 & 21472 \\
\hline prod1 & $>2854213$ & 2317393 & 89293 & 10644 & 72890 & 63674 & 64186 & 14670 & 69992 & 65679 & 62689 & 15609 & 106369 \\
\hline $\mathrm{bc1}$ & 33631 & 34127 & 40781 & 2981 & 42882 & 35132 & 25196 & 3666 & 40212 & 35132 & 25196 & 3603 & 18463 \\
\hline bienst1 & 66099 & 58841 & 19418 & 3687 & 13594 & 10427 & 13911 & 5274 & 13602 & 9248 & 9951 & 5340 & 12963 \\
\hline neos2 & $>640712$ & $>703794$ & > 609109 & 618 & 195730 & 187331 & 22742 & 4405 & 244292 & 83146 & 30790 & 2685 & 158240 \\
\hline neos3 & $>403674$ & $>393853$ & $>446035$ & 1402 & $>505184$ & $>737254$ & 556835 & 13215 & > 536938 & $>736396$ & 626894 & 15306 & 606168 \\
\hline neos7 & $>471073$ & $>339479$ & 390910 & $>44061$ & $>546623$ & 535586 & 202482 & $>55369$ & > 598209 & 498573 & 252766 & $>55799$ & 422264 \\
\hline swath1 & 33021 & 81309 & 19924 & 8285 & 64320 & 36264 & 35161 & 11268 & 73512 & 66995 & 10615 & 16471 & 128017 \\
\hline swath2 & 83441 & $>240136$ & 211976 & 22002 & 71595 & 278413 & 28808 & $>44510$ & 162695 & 258934 & 85510 & $>36277$ & 390480 \\
\hline Total (24) & 21246277 & 19421589 & 9381001 & 929347 & 8251942 & 9000334 & 7937968 & 1325589 & 8332543 & 8663537 & 7813409 & 1294569 & 10467429 \\
\hline Geom. Mean & 275789 & 262369 & 88707 & 7242 & 65966 & 70014 & 48773 & 11639.5 & 69489 & 69501 & 48377 & 12714.7 & 79269 \\
\hline
\end{tabular}


Table 3. Time in seconds needed to solve each problem instance

\begin{tabular}{|c|c|c|c|c|c|c|c|c|c|c|c|c|c|}
\hline \multirow[b]{2}{*}{ Example } & \multirow[b]{2}{*}{ random } & \multirow[b]{2}{*}{ most inf. } & \multirow[b]{2}{*}{ pscost } & \multirow[b]{2}{*}{ full str. } & \multicolumn{4}{|c|}{ lookahead $=4$} & \multicolumn{4}{|c|}{ lookahead $=8$} & \multirow{2}{*}{$\begin{array}{l}\text { CPLEX } 9.0 \\
\text { (SIP cuts) }\end{array}$} \\
\hline & & & & & $\mathrm{str} / \mathrm{ps}(10)$ & reli(1) & reli(8) & strong & $\mathrm{str} / \mathrm{ps}(10)$ & reli(1) & reli(8) & strong & \\
\hline aflow30a & $>3600.0$ & $>3600.0$ & 582.5 & 2212.6 & 364.6 & 403.6 & 416.4 & 704.7 & 457.6 & 391.7 & 368.2 & 1522.8 & 330.6 \\
\hline cap6000 & 57.1 & 50.3 & 52.6 & 47.4 & 41.1 & 38.6 & 36.1 & 58.9 & 40.7 & 37.8 & 36.3 & 59.5 & 18.8 \\
\hline gesa2-o & $>3600.0$ & $>3600.0$ & 442.7 & 864.2 & 275.0 & 203.2 & 199.1 & 463.7 & 272.5 & 284.3 & 195.7 & 926.6 & 93.9 \\
\hline mas74 & $>2651.9$ & $>2683.5$ & 2734.2 & 3087.1 & 2750.3 & 3015.9 & 2807.4 & $>3600.0$ & 2902.2 & 3066.4 & 3104.2 & $>3600.0$ & 1995.3 \\
\hline mas76 & 1307.1 & 916.0 & 291.7 & 486.6 & 274.5 & 240.6 & 225.1 & 420.2 & 315.3 & 242.2 & 161.2 & 713.9 & 136.8 \\
\hline $\operatorname{misc} 07$ & 123.4 & 54.8 & 54.6 & 299.3 & 141.6 & 97.5 & 150.2 & 213.3 & 143.2 & 113.2 & 150.2 & 267.4 & 225.6 \\
\hline pk1 & 501.3 & 483.7 & 249.9 & 488.5 & 154.3 & 199.2 & 193.3 & 658.1 & 157.2 & 200.8 & 170.3 & 885.0 & 161.7 \\
\hline pp08aCUTS & 6.8 & 7.7 & 3.4 & 12.4 & 7.8 & 4.5 & 5.4 & 10.3 & 10.3 & 4.4 & 5.5 & 9.9 & 2.4 \\
\hline qiu & $>3600.0$ & $>3600.0$ & 332.3 & $>3600.0$ & 927.4 & 369.5 & 419.1 & 1901.9 & 1213.9 & 371.2 & 341.9 & $>3600.0$ & 152.2 \\
\hline rout & $>3600.0$ & $>3600.0$ & 976.9 & 621.2 & 150.0 & 135.7 & 58.4 & 254.7 & 165.2 & 122.2 & 81.3 & 785.8 & $>3600.0$ \\
\hline vpm2 & 124.5 & 62.6 & 30.6 & 43.5 & 18.2 & 18.6 & 15.7 & 25.4 & 19.1 & 25.0 & 16.3 & 30.9 & 4.1 \\
\hline $\operatorname{ran} 8 \times 32$ & 592.4 & 1790.9 & 78.7 & 212.1 & 62.4 & 54.7 & 38.3 & 141.8 & 59.3 & 63.7 & 47.2 & 133.0 & 76.2 \\
\hline $\operatorname{ran} 10 \times 26$ & $>3600.0$ & $>3600.0$ & 192.5 & 463.0 & 89.0 & 99.5 & 86.5 & 183.1 & 100.3 & 104.7 & 82.0 & 294.2 & 113.0 \\
\hline $\operatorname{ran} 12 \times 21$ & $>3600.0$ & $>3600.0$ & 412.4 & 757.1 & 208.9 & 272.5 & 214.5 & 317.4 & 222.2 & 250.8 & 199.0 & 529.3 & 419.1 \\
\hline $\operatorname{ran} 13 \times 13$ & 1189.5 & 1168.8 & 217.7 & 287.4 & 132.8 & 159.9 & 136.9 & 233.7 & 134.4 & 144.8 & 136.1 & 355.0 & 126.9 \\
\hline mas284 & 146.4 & 116.5 & 24.6 & 59.5 & 28.5 & 28.8 & 25.9 & 48.5 & 27.8 & 23.7 & 24.1 & 71.0 & 15.4 \\
\hline prod1 & $>3600.0$ & 1741.5 & 151.0 & 405.1 & 112.9 & 94.6 & 100.8 & 287.1 & 101.1 & 96.2 & 97.8 & 359.3 & 185.7 \\
\hline bc1 & 1243.8 & 1317.7 & 1218.5 & 1180.5 & 1337.0 & 1272.9 & 1120.0 & 1108.2 & 1282.2 & 1295.2 & 1153.0 & 1071.5 & 1947.4 \\
\hline bienst1 & 868.2 & 749.2 & 230.9 & 243.0 & 121.3 & 107.9 & 115.3 & 256.8 & 138.4 & 83.3 & 91.0 & 270.3 & 194.1 \\
\hline neos2 & $>3600.0$ & $>3600.0$ & $>3600.0$ & 872.8 & 693.6 & 675.6 & 206.7 & 277.2 & 883.6 & 385.5 & 222.2 & 216.9 & 671.4 \\
\hline neos3 & $>3600.0$ & $>3600.0$ & $>3600.2$ & 2882.3 & $>3600.0$ & $>3600.0$ & 2707.2 & 1284.6 & $>3600.0$ & $>3600.0$ & 3145.8 & 1550.2 & 2513.8 \\
\hline neos7 & $>3600.0$ & $>3600.0$ & 2009.4 & $>3600.0$ & $>3600.0$ & 2661.3 & 1039.8 & $>3600.0$ & $>3600.0$ & 2377.6 & 1350.3 & $>3600.0$ & 1831.7 \\
\hline swath1 & 330.3 & 894.3 & 173.4 & 795.2 & 566.0 & 335.3 & 407.3 & 777.6 & 668.6 & 604.6 & 166.2 & 1166.1 & 693.1 \\
\hline swath2 & 1059.4 & $>3600.0$ & 2285.3 & 2876.3 & 842.3 & 3109.8 & 407.2 & $>3600.0$ & 1847.6 & 2864.5 & 1034.6 & $>3600.0$ & 3108.2 \\
\hline Total (24) & 46202.2 & 48037.5 & 19945.8 & 26397.2 & 16499.4 & 17199.6 & 11132.7 & 20427.0 & 18362.5 & 16753.8 & 12380.2 & 25618.6 & 18617.4 \\
\hline Geom. Mean & 923.6 & 938.0 & 283.4 & $\quad 504.4$ & 229.6 & 216.4 & $\quad 170.5$ & 353.2 & 253.9 & 217.2 & $\quad 170.2$ & $\quad 468.0$ & 215.8 \\
\hline
\end{tabular}


Table 4. strong branching evaluations performed and maximal depth of random branching

\begin{tabular}{|c|c|c|c|c|c|c|c|c|c|c|}
\hline \multirow[b]{2}{*}{ Example } & \multirow[b]{2}{*}{ full str. } & \multicolumn{4}{|c|}{ lookahead $=4$} & \multicolumn{4}{|c|}{ lookahead $=8$} & \multirow{2}{*}{$\begin{array}{l}\text { avg. depth } \\
\text { full str. }\end{array}$} \\
\hline & & str/ps(10) & reli(1) & reli(8) & strong & str/ps(10) & reli(1) & reli(8) & strong & \\
\hline aflow30a & 972871 & 3625 & 396 & 2847 & 239787 & 5604 & 397 & 2865 & 495934 & 38.7 \\
\hline cap6000 & 4602 & 762 & 92 & 597 & 4683 & 762 & 92 & 597 & 4683 & 16.7 \\
\hline gesa2-o & 401182 & 2231 & 435 & 2331 & 114464 & 3373 & 1841 & 2178 & 235608 & 28.8 \\
\hline mas74 & 8773236 & 6609 & 98 & 649 & 6094848 & 10531 & 88 & 673 & 6617267 & 23.3 \\
\hline mas76 & 1508479 & 6307 & 69 & 530 & 861005 & 9080 & 69 & 558 & 1551253 & 21.2 \\
\hline $\operatorname{misc} 07$ & 85451 & 3120 & 379 & 2471 & 65988 & 2943 & 266 & 3262 & 84665 & 29.9 \\
\hline pk1 & 897902 & 7008 & 54 & 391 & 879768 & 9334 & 54 & 402 & 1256800 & 24.6 \\
\hline pp08aCUTS & 2227 & 954 & 61 & 433 & 1498 & 1531 & 60 & 438 & 1530 & 7.3 \\
\hline qiu & 79685 & 6879 & 48 & 363 & 47170 & 11732 & 48 & 370 & 87289 & 11.6 \\
\hline rout & 114383 & 3004 & 573 & 2703 & 58916 & 5554 & 426 & 3473 & 210641 & 22.1 \\
\hline vpm2 & 21665 & 1948 & 165 & 881 & 12712 & 2971 & 141 & 1089 & 15435 & 18.0 \\
\hline ran8x32 & 129698 & 1770 & 570 & 2657 & 63719 & 2765 & 667 & 2534 & 58732 & 24.8 \\
\hline $\operatorname{ran} 10 \times 26$ & 267261 & 3038 & 536 & 1985 & 88240 & 3771 & 586 & 3417 & 144009 & 30.2 \\
\hline $\operatorname{ran} 12 \times 21$ & 456400 & 5414 & 593 & 2675 & 141880 & 6588 & 940 & 2591 & 269767 & 25.5 \\
\hline $\operatorname{ran} 13 \times 13$ & 210862 & 5304 & 167 & 1276 & 144070 & 7920 & 311 & 1521 & 234413 & 26.3 \\
\hline mas284 & 54311 & 4350 & 110 & 462 & 32685 & 5010 & 85 & 477 & 47686 & 14.9 \\
\hline prod1 & 209540 & 151 & 153 & 1202 & 118575 & 335 & 158 & 1173 & 152860 & 32.9 \\
\hline bc1 & 17413 & 804 & 3189 & 5092 & 16891 & 787 & 3189 & 5092 & 16810 & 35.9 \\
\hline bienst1 & 31222 & 4150 & 27 & 211 & 27841 & 4380 & 27 & 206 & 30331 & 13.8 \\
\hline neos2 & 115097 & 1076 & 9998 & 15893 & 44008 & 267 & 13311 & 13994 & 37362 & 121.6 \\
\hline neos3 & 305325 & 1264 & 11730 & 45143 & 170679 & 270 & 19218 & 54459 & 223816 & 134.6 \\
\hline neos7 & 475374 & 1058 & 6170 & 11256 & 344673 & 171 & 5944 & 17062 & 417934 & 130.2 \\
\hline swath1 & 111797 & 1682 & 1598 & 8170 & 80944 & 3235 & 2088 & 6474 & 129431 & 28.1 \\
\hline swath2 & 323669 & 2304 & 1915 & 7425 & 310410 & 3168 & 3551 & 8640 & 327248 & 38.0 \\
\hline Total (24) & 15569652 & 74812 & 39126 & 117643 & 9965454 & 102082 & 53557 & 133545 & 12651504 & - \\
\hline Geom. Mean & 146784 & 2284 & 375 & 1850 & 86189 & 2524 & 429 & 1998 & 119799 & 28.2 \\
\hline
\end{tabular}




\begin{tabular}{lrrrrrrr}
\hline Example & B \& B & Cuts & StrBra & Dual Bound & Primal Bound & Time & Gap \% \\
\hline aflow30a & 1248221 & 22 & 0 & 1121.680021 & 1180 & 3600.0 & 5.199 \\
cap6000 & 7454 & 3 & 0 & -2451377 & -2451377 & 57.1 & 0.000 \\
gesa2-o & 1023765 & 171 & 0 & 25636165.22 & 25804886.59 & 3600.0 & 0.658 \\
mas74 & 4662991 & 0 & 0 & 11316.99594 & 12156.87628 & 2651.9 & 7.421 \\
mas76 & 2935689 & 0 & 0 & 40005.05414 & 40005.05414 & 1307.1 & 0.000 \\
misc07 & 47941 & 71 & 0 & 2810 & 2810 & 123.4 & 0.000 \\
pk1 & 1064666 & 0 & 0 & 11 & 11 & 501.3 & 0.000 \\
pp08aCUTS & 1877 & 494 & 0 & 7350 & 7350 & 6.8 & 0.000 \\
qiu & 163998 & 1 & 0 & -183.4303822 & -132.8731378 & 3600.0 & 27.562 \\
rout & 714812 & 68 & 0 & 1026.780486 & 1079.19 & 3600.0 & 5.104 \\
vpm2 & 92880 & 214 & 0 & 13.75 & 13.75 & 124.5 & 0.000 \\
ran8x32 & 321933 & 93 & 0 & 5247 & 5247 & 592.4 & 0.000 \\
ran10x26 & 1637058 & 56 & 0 & 4191.797533 & 4290 & 3600.0 & 2.343 \\
ran12x21 & 1667430 & 62 & 0 & 3626.01421 & 3677 & 3600.0 & 1.406 \\
ran13x13 & 907443 & 78 & 0 & 3252 & 3252 & 1189.5 & 0.000 \\
mas284 & 162255 & 0 & 0 & 91405.72368 & 91405.72368 & 146.4 & 0.000 \\
prod1 & 2854213 & 133 & 0 & -60.80537903 & -56 & 3600.0 & 7.903 \\
bc1 & 33631 & 0 & 0 & 3.338362548 & 3.338362548 & 1243.8 & 0.000 \\
bienst1 & 66099 & 23 & 0 & 46.75 & 46.75 & 868.2 & 0.000 \\
neos2 & 640712 & 29 & 0 & -192.8700538 & 486.920203 & 3600.1 & 352.460 \\
neos3 & 403674 & 42 & 0 & -2300.094909 & 480.6941847 & 3600.0 & 120.899 \\
neos7 & 471073 & 112 & 0 & 666536.746 & 727934 & 3600.0 & 9.211 \\
swath1 & 33021 & 59 & 0 & 379.0712957 & 379.0712957 & 330.3 & 0.000 \\
swath2 & 83441 & 80 & 0 & 385.1996929 & 385.1996929 & 1059.4 & 0.000 \\
\hline Total (24) & 21246277 & 1811 & 0 & & & 46202.3 & 540.167 \\
Geom. Mean & 275789.4 & & 0.0 & & & 923.6 & \\
\hline & & & & & & &
\end{tabular}

Table 5: random branching 


\begin{tabular}{lrrrrrrr}
\hline Example & B \& B & Cuts & StrBra & Dual Bound & Primal Bound & Time & Gap \% \\
\hline aflow30a & 1025389 & 22 & 0 & 1128.084312 & 1182 & 3600.0 & 4.779 \\
cap6000 & 6717 & 3 & 0 & -2451377 & -2451377 & 50.3 & 0.000 \\
gesa2-o & 1150438 & 171 & 0 & 25695246.64 & 25799147.33 & 3600.0 & 0.404 \\
mas74 & 4725208 & 0 & 0 & 11328.45882 & 12199.33728 & 2683.5 & 7.688 \\
mas76 & 2038945 & 0 & 0 & 40005.05414 & 40005.05414 & 916.0 & 0.000 \\
misc07 & 17955 & 71 & 0 & 2810 & 2810 & 54.8 & 0.000 \\
pk1 & 850198 & 0 & 0 & 11 & 11 & 483.7 & 0.000 \\
pp08aCUTS & 1740 & 494 & 0 & 7350 & 7350 & 7.7 & 0.000 \\
qiu & 165538 & 1 & 0 & -173.0330337 & -132.873137 & 3600.0 & 23.209 \\
rout & 702392 & 68 & 0 & 1018.80106 & 1083.54 & 3600.0 & 6.354 \\
vpm2 & 40258 & 214 & 0 & 13.75 & 13.75 & 62.6 & 0.000 \\
ran8x32 & 674613 & 93 & 0 & 5247 & 5247 & 1790.9 & 0.000 \\
ran10x26 & 1500171 & 56 & 0 & 4197.825257 & 4281 & 3600.0 & 1.981 \\
ran12x21 & 1471340 & 62 & 0 & 3633.577589 & 3664 & 3600.0 & 0.837 \\
ran13x13 & 758186 & 78 & 0 & 3252 & 3252 & 1168.8 & 0.000 \\
mas284 & 123569 & 0 & 0 & 91405.72368 & 91405.72368 & 116.5 & 0.000 \\
prod1 & 2317393 & 133 & 0 & -56 & -56 & 1741.5 & 0.000 \\
bc1 & 34127 & 0 & 0 & 3.338362548 & 3.338362548 & 1317.7 & 0.000 \\
bienst1 & 58841 & 23 & 0 & 46.75 & 46.75 & 749.2 & 0.000 \\
neos2 & 703794 & 29 & 0 & -349.441453 & 513.2718699 & 3600.0 & 246.884 \\
neos3 & 393853 & 42 & 0 & -1511.754003 & 490.5778177 & 3600.0 & 132.451 \\
neos7 & 339479 & 112 & 0 & 650826.8103 & 733934 & 3600.0 & 12.769 \\
swath1 & 81309 & 59 & 0 & 379.0712957 & 379.0712957 & 894.3 & 0.000 \\
swath2 & 240136 & 80 & 0 & 382.724 & 385.1996929 & 3600.0 & 0.647 \\
\hline Total (24) & 19421589 & 1811 & 0 & & & 48037.5 & 438.004 \\
Geom. Mean & 262368.9 & & 0.0 & & & 938.0 & \\
\hline & & & & & & &
\end{tabular}

Table 6: most infeasible branching 


\begin{tabular}{lrrrrrrr}
\hline Example & B \& B & Cuts & StrBra & Dual Bound & Primal Bound & Time & Gap \% \\
\hline aflow30a & 46649 & 22 & 973061 & 1158 & 1158 & 2229.9 & 0.000 \\
cap6000 & 3402 & 3 & 4602 & -2451377 & -2451377 & 47.1 & 0.000 \\
gesa2-o & 13743 & 171 & 437133 & 25779856.37 & 25779856.37 & 856.6 & 0.000 \\
mas74 & 551780 & 0 & 9853577 & 11801.18573 & 11801.18573 & 3306.4 & 0.000 \\
mas76 & 114228 & 0 & 1686361 & 40005.05414 & 40005.05414 & 489.4 & 0.000 \\
misc07 & 2713 & 71 & 95342 & 2810 & 2810 & 292.4 & 0.000 \\
pk1 & 56546 & 0 & 996973 & 11 & 11 & 505.2 & 0.000 \\
pp08aCUTS & 124 & 494 & 2309 & 7350 & 7350 & 12.0 & 0.000 \\
qiu & 15659 & 1 & 361712 & -132.8731853 & -132.8731853 & 11133.0 & 0.000 \\
rout & 2836 & 68 & 126592 & 1077.56 & 1077.56 & 625.7 & 0.000 \\
vpm2 & 1457 & 214 & 23490 & 13.75 & 13.75 & 44.1 & 0.000 \\
ran8x32 & 4902 & 93 & 134873 & 5247 & 5247 & 214.4 & 0.000 \\
ran10x26 & 8520 & 56 & 283797 & 4270 & 4270 & 471.1 & 0.000 \\
ran12x21 & 13420 & 62 & 486298 & 3664 & 3664 & 780.0 & 0.000 \\
ran13x13 & 9147 & 78 & 226258 & 3252 & 3252 & 289.5 & 0.000 \\
mas284 & 3226 & 0 & 57953 & 91405.72368 & 91405.72368 & 61.2 & 0.000 \\
prod1 & 10644 & 143 & 226101 & -56 & -56 & 401.9 & 0.000 \\
bc1 & 2981 & 0 & 18028 & 3.338362548 & 3.338362548 & 1194.1 & 0.000 \\
bienst1 & 3687 & 23 & 32384 & 46.75 & 46.75 & 237.8 & 0.000 \\
neos2 & 618 & 32 & 115613 & 454.864697 & 454.864697 & 872.3 & 0.000 \\
neos3 & 1402 & 42 & 309856 & 368.842751 & 368.842751 & 2967.0 & 0.000 \\
neos7 & 476601 & 112 & 4079187 & 721934 & 721934 & 33417.8 & 0.000 \\
swath1 & 8285 & 59 & 122776 & 379.0712957 & 379.0712957 & 801.7 & 0.000 \\
swath2 & 22002 & 80 & 361767 & 385.1996929 & 385.1996929 & 2852.2 & 0.000 \\
\hline Total (24) & 1374572 & 1824 & 21016043 & & & 64102.9 & 0.000 \\
Geom. Mean & 8570.1 & & 181371.7 & & & 584.2 & \\
\hline & & & & & &
\end{tabular}

Table 7: full strong branching 


\begin{tabular}{lrrrrrrr}
\hline Example & B \& B & Cuts & StrBra & Dual Bound & Primal Bound & Time & Gap \% \\
\hline aflow30a & 276002 & 22 & 0 & 1158 & 1158 & 582.5 & 0.000 \\
cap6000 & 6791 & 3 & 0 & -2451377 & -2451377 & 52.6 & 0.000 \\
gesa2-o & 126459 & 171 & 0 & 25779856.37 & 25779856.37 & 442.7 & 0.000 \\
mas74 & 5160828 & 0 & 0 & 11801.18573 & 11801.18573 & 2734.2 & 0.000 \\
mas76 & 603683 & 0 & 0 & 40005.05414 & 40005.05414 & 291.7 & 0.000 \\
misc07 & 19407 & 71 & 0 & 2810 & 2810 & 54.6 & 0.000 \\
pk1 & 437758 & 0 & 0 & 11 & 11 & 249.9 & 0.000 \\
pp08aCUTS & 673 & 494 & 0 & 7350 & 7350 & 3.4 & 0.000 \\
qiu & 15471 & 1 & 0 & -132.873137 & -132.873137 & 332.3 & 0.000 \\
rout & 309779 & 68 & 0 & 1077.56 & 1077.56 & 976.9 & 0.000 \\
vpm2 & 22568 & 214 & 0 & 13.75 & 13.75 & 30.6 & 0.000 \\
ran8x32 & 40069 & 93 & 0 & 5247 & 5247 & 78.7 & 0.000 \\
ran10x26 & 128327 & 56 & 0 & 4270 & 4270 & 192.5 & 0.000 \\
ran12x21 & 234915 & 62 & 0 & 3664 & 3664 & 412.4 & 0.000 \\
ran13x13 & 149239 & 78 & 0 & 3252 & 3252 & 217.7 & 0.000 \\
mas284 & 21586 & 0 & 0 & 91405.72368 & 91405.72368 & 24.6 & 0.000 \\
prod1 & 89293 & 133 & 0 & -56 & -56 & 151.0 & 0.000 \\
bc1 & 40781 & 0 & 0 & 3.338362548 & 3.338362548 & 1218.5 & 0.000 \\
bienst1 & 19418 & 23 & 0 & 46.75 & 46.75 & 230.9 & 0.000 \\
neos2 & 609109 & 29 & 0 & 131.4396174 & 499.0922328 & 3600.0 & 279.712 \\
neos3 & 446035 & 42 & 0 & -1525.807871 & 505.4519055 & 3600.2 & 133.127 \\
neos7 & 390910 & 112 & 0 & 721934 & 721934 & 2009.4 & 0.000 \\
swath1 & 19924 & 59 & 0 & 379.0712957 & 379.0712957 & 173.4 & 0.000 \\
swath2 & 211976 & 80 & 0 & 385.1996929 & 385.1996929 & 2285.3 & 0.000 \\
\hline Total (24) & 9381001 & 1811 & 0 & & & 19945.8 & 412.839 \\
Geom. Mean & 88706.8 & & 0.0 & & & 283.4 & \\
\hline & & & & & & &
\end{tabular}

Table 8: pseudocost branching 


\begin{tabular}{lrrrrrrr}
\hline Example & B \& B & Cuts & StrBra & Dual Bound & Primal Bound & Time & Gap \% \\
\hline aflow30a & 203544 & 22 & 396 & 1158 & 1158 & 403.6 & 0.000 \\
cap6000 & 5076 & 3 & 92 & -2451377 & -2451377 & 38.6 & 0.000 \\
gesa2-o & 57534 & 171 & 435 & 25779856.37 & 25779856.37 & 203.2 & 0.000 \\
mas74 & 5478830 & 0 & 98 & 11801.18573 & 11801.18573 & 3015.9 & 0.000 \\
mas76 & 496370 & 0 & 69 & 40005.05414 & 40005.05414 & 240.6 & 0.000 \\
misc07 & 35187 & 71 & 379 & 2810 & 2810 & 97.5 & 0.000 \\
pk1 & 367763 & 0 & 54 & 11 & 11 & 199.2 & 0.000 \\
pp08aCUTS & 673 & 494 & 61 & 7350 & 7350 & 4.5 & 0.000 \\
qiu & 16479 & 1 & 48 & -132.8731612 & -132.8731612 & 369.5 & 0.000 \\
rout & 44232 & 68 & 573 & 1077.56 & 1077.56 & 135.7 & 0.000 \\
vpm2 & 13780 & 214 & 165 & 13.75 & 13.75 & 18.6 & 0.000 \\
ran8x32 & 26360 & 93 & 570 & 5247 & 5247 & 54.7 & 0.000 \\
ran10x26 & 64640 & 56 & 536 & 4270 & 4270 & 99.5 & 0.000 \\
ran12x21 & 172161 & 62 & 593 & 3664 & 3664 & 272.5 & 0.000 \\
ran13x13 & 109241 & 78 & 167 & 3252 & 3252 & 159.9 & 0.000 \\
mas284 & 24383 & 0 & 110 & 91405.72368 & 91405.72368 & 28.8 & 0.000 \\
prod1 & 63674 & 167 & 153 & -56 & -56 & 94.6 & 0.000 \\
bc1 & 35132 & 0 & 3189 & 3.338362548 & 3.338362548 & 1272.9 & 0.000 \\
bienst1 & 10427 & 23 & 27 & 46.75 & 46.75 & 107.9 & 0.000 \\
neos2 & 187331 & 29 & 9998 & 454.864697 & 454.864697 & 675.6 & 0.000 \\
neos3 & 737254 & 42 & 11730 & 229.611825 & 414.5387268 & 3600.0 & 80.539 \\
neos7 & 535586 & 112 & 6170 & 721934 & 721934 & 2661.3 & 0.000 \\
swath1 & 36264 & 59 & 1598 & 379.0712957 & 379.0712957 & 335.3 & 0.000 \\
swath2 & 278413 & 80 & 1915 & 385.1996929 & 385.1996929 & 3109.8 & 0.000 \\
\hline Total (24) & 9000334 & 1845 & 39126 & & & 17199.6 & 80.539 \\
Geom. Mean & 70013.6 & & 374.8 & & & 216.4 & \\
\hline & & & & & &
\end{tabular}

Table 9: reliability branching $\left(\eta_{\text {rel }}=1, \lambda=4\right)$ 


\begin{tabular}{lrrrrrrr}
\hline Example & B \& B & Cuts & StrBra & Dual Bound & Primal Bound & Time & Gap \% \\
\hline aflow30a & 180019 & 22 & 1453 & 1158 & 1158 & 357.6 & 0.000 \\
cap6000 & 4474 & 3 & 331 & -2451377 & -2451377 & 35.1 & 0.000 \\
gesa2-o & 55888 & 171 & 1486 & 25779856.37 & 25779856.37 & 203.2 & 0.000 \\
mas74 & 3797950 & 0 & 316 & 11801.18573 & 11801.18573 & 1841.2 & 0.000 \\
mas76 & 487445 & 0 & 302 & 40005.05414 & 40005.05414 & 234.5 & 0.000 \\
misc07 & 54541 & 71 & 1178 & 2810 & 2810 & 141.5 & 0.000 \\
pk1 & 345480 & 0 & 204 & 11 & 11 & 197.9 & 0.000 \\
pp08aCUTS & 434 & 494 & 232 & 7350 & 7350 & 4.0 & 0.000 \\
qiu & 18619 & 1 & 187 & -132.8731399 & -132.8731399 & 432.2 & 0.000 \\
rout & 25038 & 68 & 1615 & 1077.56 & 1077.56 & 88.5 & 0.000 \\
vpm2 & 12502 & 214 & 394 & 13.75 & 13.75 & 17.5 & 0.000 \\
ran8x32 & 26659 & 93 & 1660 & 5247 & 5247 & 53.9 & 0.000 \\
ran10x26 & 52969 & 56 & 1227 & 4270 & 4270 & 89.2 & 0.000 \\
ran12x21 & 137797 & 62 & 2717 & 3664 & 3664 & 238.1 & 0.000 \\
ran13x13 & 94288 & 78 & 842 & 3252 & 3252 & 120.2 & 0.000 \\
mas284 & 21146 & 0 & 244 & 91405.72368 & 91405.72368 & 24.5 & 0.000 \\
prod1 & 61355 & 167 & 699 & -56 & -56 & 94.4 & 0.000 \\
bc1 & 28159 & 0 & 4921 & 3.338362548 & 3.338362548 & 1127.6 & 0.000 \\
bienst1 & 12603 & 23 & 108 & 46.75 & 46.75 & 131.5 & 0.000 \\
neos2 & 14779 & 29 & 8088 & 454.864697 & 454.864697 & 131.0 & 0.000 \\
neos3 & 820037 & 42 & 47044 & 368.842751 & 368.842751 & 3573.0 & 0.000 \\
neos7 & 574309 & 112 & 24899 & 721934 & 721934 & 3329.3 & 0.000 \\
swath1 & 22776 & 59 & 5016 & 379.0712957 & 379.0712957 & 257.4 & 0.000 \\
swath2 & 57431 & 80 & 5465 & 385.1996929 & 385.1996929 & 679.8 & 0.000 \\
\hline Total (24) & 6906698 & 1845 & 110628 & & & 13402.9 & 0.000 \\
Geom. Mean & 53522.9 & & 1176.7 & & & 178.2 & \\
\hline & & & & & &
\end{tabular}

Table 10: reliability branching $\left(\eta_{\mathrm{rel}}=4, \lambda=4\right)$ 


\begin{tabular}{lrrrrrrr}
\hline Example & B \& B & Cuts & StrBra & Dual Bound & Primal Bound & Time & Gap \% \\
\hline aflow30a & 181397 & 22 & 2847 & 1158 & 1158 & 416.4 & 0.000 \\
cap6000 & 4253 & 3 & 597 & -2451377 & -2451377 & 36.1 & 0.000 \\
gesa2-o & 53881 & 171 & 2331 & 25779856.37 & 25779856.37 & 199.1 & 0.000 \\
mas74 & 5521061 & 0 & 649 & 11801.18573 & 11801.18573 & 2807.4 & 0.000 \\
mas76 & 482122 & 0 & 530 & 40005.05414 & 40005.05414 & 225.1 & 0.000 \\
misc07 & 54932 & 71 & 2471 & 2810 & 2810 & 150.2 & 0.000 \\
pk1 & 331339 & 0 & 391 & 11 & 11 & 193.3 & 0.000 \\
pp08aCUTS & 489 & 494 & 433 & 7350 & 7350 & 5.4 & 0.000 \\
qiu & 18405 & 1 & 363 & -132.8731399 & -132.8731399 & 419.1 & 0.000 \\
rout & 13883 & 68 & 2703 & 1077.56 & 1077.56 & 58.4 & 0.000 \\
vpm2 & 9648 & 214 & 881 & 13.75 & 13.75 & 15.7 & 0.000 \\
ran8x32 & 17668 & 93 & 2657 & 5247 & 5247 & 38.3 & 0.000 \\
ran10x26 & 48065 & 56 & 1985 & 4270 & 4270 & 86.5 & 0.000 \\
ran12x21 & 135037 & 62 & 2675 & 3664 & 3664 & 214.5 & 0.000 \\
ran13x13 & 95288 & 78 & 1276 & 3252 & 3252 & 136.9 & 0.000 \\
mas284 & 21179 & 0 & 462 & 91405.72368 & 91405.72368 & 25.9 & 0.000 \\
prod1 & 64186 & 167 & 1202 & -56 & -56 & 100.8 & 0.000 \\
bc1 & 25196 & 0 & 5092 & 3.338362548 & 3.338362548 & 1120.0 & 0.000 \\
bienst1 & 13911 & 23 & 211 & 46.75 & 46.75 & 115.3 & 0.000 \\
neos2 & 22742 & 29 & 15893 & 454.864697 & 454.864697 & 206.7 & 0.000 \\
neos3 & 556835 & 42 & 45143 & 368.842751 & 368.842751 & 2707.2 & 0.000 \\
neos7 & 202482 & 112 & 11256 & 721934 & 721934 & 1039.8 & 0.000 \\
swath1 & 35161 & 59 & 8170 & 379.0712957 & 379.0712957 & 407.3 & 0.000 \\
swath2 & 28808 & 80 & 7425 & 385.1996929 & 385.1996929 & 407.2 & 0.000 \\
\hline Total (24) & 7937968 & 1845 & 117643 & & & 11132.7 & 0.000 \\
Geom. Mean & 48772.8 & & 1850.3 & & & 170.5 & \\
\hline & & & & & & &
\end{tabular}

Table 11: reliability branching $\left(\eta_{\mathrm{rel}}=8, \lambda=4\right)$ 


\begin{tabular}{lrrrrrrr}
\hline Example & B \& B & Cuts & StrBra & Dual Bound & Primal Bound & Time & Gap \% \\
\hline aflow30a & 175649 & 22 & 5554 & 1158 & 1158 & 426.2 & 0.000 \\
cap6000 & 4998 & 3 & 1099 & -2451377 & -2451377 & 46.6 & 0.000 \\
gesa2-o & 51495 & 171 & 3800 & 25779856.37 & 25779856.37 & 203.7 & 0.000 \\
mas74 & 3803361 & 0 & 1173 & 11801.18573 & 11801.18573 & 1848.8 & 0.000 \\
mas76 & 497653 & 0 & 1042 & 40005.05414 & 40005.05414 & 246.0 & 0.000 \\
misc07 & 38680 & 71 & 6352 & 2810 & 2810 & 115.1 & 0.000 \\
pk1 & 313777 & 0 & 756 & 11 & 11 & 176.5 & 0.000 \\
pp08aCUTS & 324 & 494 & 896 & 7350 & 7350 & 7.0 & 0.000 \\
qiu & 13101 & 1 & 723 & -132.8731399 & -132.8731399 & 323.7 & 0.000 \\
rout & 19412 & 68 & 5339 & 1077.56 & 1077.56 & 90.2 & 0.000 \\
vpm2 & 9870 & 214 & 1974 & 13.75 & 13.75 & 17.4 & 0.000 \\
ran8x32 & 18728 & 93 & 5116 & 5247 & 5247 & 48.3 & 0.000 \\
ran10x26 & 39207 & 56 & 7289 & 4270 & 4270 & 76.3 & 0.000 \\
ran12x21 & 112818 & 62 & 6068 & 3664 & 3664 & 192.1 & 0.000 \\
ran13x13 & 97849 & 78 & 4815 & 3252 & 3252 & 155.1 & 0.000 \\
mas284 & 20815 & 0 & 931 & 91405.72368 & 91405.72368 & 25.8 & 0.000 \\
prod1 & 65130 & 167 & 2375 & -56 & -56 & 105.4 & 0.000 \\
bc1 & 24188 & 0 & 7414 & 3.338362548 & 3.338362548 & 1204.0 & 0.000 \\
bienst1 & 14586 & 23 & 409 & 46.75 & 46.75 & 139.9 & 0.000 \\
neos2 & 9370 & 29 & 14558 & 454.864697 & 454.864697 & 144.2 & 0.000 \\
neos3 & 301563 & 42 & 56269 & 368.842751 & 368.842751 & 1928.9 & 0.000 \\
neos7 & 283419 & 112 & 22956 & 721934 & 721934 & 1789.2 & 0.000 \\
swath1 & 16857 & 59 & 13103 & 379.0712957 & 379.0712957 & 297.9 & 0.000 \\
swath2 & 89174 & 80 & 17567 & 385.1996929 & 385.1996929 & 1174.3 & 0.000 \\
\hline Total (24) & 6022024 & 1845 & 187578 & & & 10782.6 & 0.000 \\
Geom. Mean & 44649.9 & & 3640.6 & & & 179.0 & \\
\hline & & & & & & &
\end{tabular}

Table 12: reliability branching $\left(\eta_{\text {rel }}=16, \lambda=4\right)$ 


\begin{tabular}{lrrrrrrr}
\hline Example & B \& B & Cuts & StrBra & Dual Bound & Primal Bound & Time & Gap \% \\
\hline aflow30a & 235478 & 22 & 11184 & 1158 & 1158 & 562.5 & 0.000 \\
cap6000 & 4010 & 3 & 1695 & -2451377 & -2451377 & 42.2 & 0.000 \\
gesa2-o & 40561 & 171 & 6902 & 25779856.37 & 25779856.37 & 175.5 & 0.000 \\
mas74 & 5961790 & 0 & 2447 & 11801.18573 & 11801.18573 & 3370.3 & 0.000 \\
mas76 & 501169 & 0 & 1940 & 40005.05414 & 40005.05414 & 241.1 & 0.000 \\
misc07 & 46618 & 71 & 11622 & 2810 & 2810 & 154.4 & 0.000 \\
pk1 & 292458 & 0 & 1494 & 11 & 11 & 157.6 & 0.000 \\
pp08aCUTS & 280 & 494 & 1422 & 7350 & 7350 & 9.9 & 0.000 \\
qiu & 14163 & 1 & 1470 & -132.8731399 & -132.8731399 & 382.1 & 0.000 \\
rout & 19346 & 68 & 9680 & 1077.56 & 1077.56 & 114.3 & 0.000 \\
vpm2 & 8959 & 214 & 3171 & 13.75 & 13.75 & 19.4 & 0.000 \\
ran8x32 & 12726 & 93 & 7783 & 5247 & 5247 & 42.9 & 0.000 \\
ran10x26 & 42754 & 56 & 8036 & 4270 & 4270 & 96.2 & 0.000 \\
ran12x21 & 116887 & 62 & 8973 & 3664 & 3664 & 207.8 & 0.000 \\
ran13x13 & 81012 & 78 & 5962 & 3252 & 3252 & 122.0 & 0.000 \\
mas284 & 20839 & 0 & 1770 & 91405.72368 & 91405.72368 & 27.2 & 0.000 \\
prod1 & 67782 & 167 & 4442 & -56 & -56 & 113.4 & 0.000 \\
bc1 & 20416 & 0 & 10331 & 3.338362548 & 3.338362548 & 1214.2 & 0.000 \\
bienst1 & 10126 & 23 & 831 & 46.75 & 46.75 & 103.6 & 0.000 \\
neos2 & 6843 & 29 & 23911 & 454.864697 & 454.864697 & 188.3 & 0.000 \\
neos3 & 167218 & 42 & 58432 & 368.842751 & 368.842751 & 1310.0 & 0.000 \\
neos7 & 221647 & 112 & 31955 & 721934 & 721934 & 1489.7 & 0.000 \\
swath1 & 20639 & 59 & 19722 & 379.0712957 & 379.0712957 & 416.1 & 0.000 \\
swath2 & 27076 & 80 & 17839 & 385.1996929 & 385.1996929 & 542.2 & 0.000 \\
\hline Total (24) & 7940797 & 1845 & 253014 & & & 11103.0 & 0.000 \\
Geom. Mean & 39655.2 & & 5837.8 & & & 184.2 & \\
\hline & & & & & & &
\end{tabular}

Table 13: reliability branching $\left(\eta_{\text {rel }}=32, \lambda=4\right)$ 


\begin{tabular}{lrrrrrrr}
\hline Example & B \& B & Cuts & StrBra & Dual Bound & Primal Bound & Time & Gap \% \\
\hline aflow30a & 144280 & 22 & 3268 & 1158 & 1158 & 297.9 & 0.000 \\
cap6000 & 4474 & 3 & 331 & -2451377 & -2451377 & 35.7 & 0.000 \\
gesa2-o & 40741 & 171 & 1816 & 25779856.37 & 25779856.37 & 152.6 & 0.000 \\
mas74 & 5348980 & 0 & 659 & 11801.18573 & 11801.18573 & 2859.8 & 0.000 \\
mas76 & 464712 & 0 & 514 & 40005.05414 & 40005.05414 & 217.6 & 0.000 \\
misc07 & 49930 & 71 & 1590 & 2810 & 2810 & 134.2 & 0.000 \\
pk1 & 367800 & 0 & 463 & 11 & 11 & 229.8 & 0.000 \\
pp08aCUTS & 324 & 494 & 524 & 7350 & 7350 & 5.1 & 0.000 \\
qiu & 14663 & 1 & 358 & -132.8731399 & -132.8731399 & 357.0 & 0.000 \\
rout & 24331 & 68 & 3397 & 1077.56 & 1077.56 & 101.0 & 0.000 \\
vpm2 & 11174 & 214 & 954 & 13.75 & 13.75 & 17.3 & 0.000 \\
ran8x32 & 25860 & 93 & 2425 & 5247 & 5247 & 57.1 & 0.000 \\
ran10x26 & 47026 & 56 & 2316 & 4270 & 4270 & 88.1 & 0.000 \\
ran12x21 & 120986 & 62 & 3124 & 3664 & 3664 & 195.1 & 0.000 \\
ran13x13 & 83928 & 78 & 1975 & 3252 & 3252 & 123.1 & 0.000 \\
mas284 & 19724 & 0 & 377 & 91405.72368 & 91405.72368 & 25.3 & 0.000 \\
prod1 & 60992 & 167 & 686 & -56 & -56 & 99.3 & 0.000 \\
bc1 & 28159 & 0 & 4921 & 3.338362548 & 3.338362548 & 1167.8 & 0.000 \\
bienst1 & 10917 & 23 & 274 & 46.75 & 46.75 & 123.2 & 0.000 \\
neos2 & 45534 & 29 & 16141 & 454.864697 & 454.864697 & 254.8 & 0.000 \\
neos3 & 760920 & 42 & 39282 & 368.842751 & 368.842751 & 3228.7 & 0.000 \\
neos7 & 332730 & 112 & 7631 & 721934 & 721934 & 1743.7 & 0.000 \\
swath1 & 13953 & 59 & 7755 & 379.0712957 & 379.0712957 & 214.9 & 0.000 \\
swath2 & 12363 & 80 & 7087 & 385.1996929 & 385.1996929 & 227.8 & 0.000 \\
\hline Total (24) & 8034501 & 1845 & 107868 & & & 11956.9 & 0.000 \\
Geom. Mean & 47082.6 & & 1775.0 & & & 169.3 & \\
\hline & & & & & &
\end{tabular}

Table 14: dynamic reliability branching $\left(\eta_{\text {rel }}=4-16, \lambda=4\right)$ 


\begin{tabular}{lrrrrrrr}
\hline Example & B \& B & Cuts & StrBra & Dual Bound & Primal Bound & Time & Gap \% \\
\hline aflow30a & 149993 & 22 & 6162 & 1158 & 1158 & 317.2 & 0.000 \\
cap6000 & 4552 & 3 & 326 & -2451377 & -2451377 & 34.8 & 0.000 \\
gesa2-o & 55411 & 171 & 2256 & 25779856.37 & 25779856.37 & 213.0 & 0.000 \\
mas74 & 4290897 & 0 & 1092 & 11801.18573 & 11801.18573 & 2094.2 & 0.000 \\
mas76 & 484027 & 0 & 824 & 40005.05414 & 40005.05414 & 217.3 & 0.000 \\
misc07 & 48698 & 71 & 2356 & 2810 & 2810 & 140.6 & 0.000 \\
pk1 & 386040 & 0 & 769 & 11 & 11 & 239.8 & 0.000 \\
pp08aCUTS & 301 & 494 & 766 & 7350 & 7350 & 6.2 & 0.000 \\
qiu & 14177 & 1 & 554 & -132.8731517 & -132.8731517 & 339.2 & 0.000 \\
rout & 14769 & 68 & 5986 & 1077.56 & 1077.56 & 86.5 & 0.000 \\
vpm2 & 10698 & 214 & 1607 & 13.75 & 13.75 & 18.8 & 0.000 \\
ran8x32 & 19384 & 93 & 4884 & 5247 & 5247 & 48.6 & 0.000 \\
ran10x26 & 42580 & 56 & 4466 & 4270 & 4270 & 85.0 & 0.000 \\
ran12x21 & 126350 & 62 & 4788 & 3664 & 3664 & 211.6 & 0.000 \\
ran13x13 & 82494 & 78 & 3580 & 3252 & 3252 & 110.1 & 0.000 \\
mas284 & 21934 & 0 & 571 & 91405.72368 & 91405.72368 & 28.4 & 0.000 \\
prod1 & 63495 & 167 & 755 & -56 & -56 & 100.2 & 0.000 \\
bc1 & 28159 & 0 & 4921 & 3.338362548 & 3.338362548 & 1177.5 & 0.000 \\
bienst1 & 13603 & 23 & 463 & 46.75 & 46.75 & 149.5 & 0.000 \\
neos2 & 24153 & 29 & 13343 & 454.864697 & 454.864697 & 192.1 & 0.000 \\
neos3 & 546419 & 42 & 56572 & 368.842751 & 368.842751 & 2602.5 & 0.000 \\
neos7 & 252863 & 112 & 18920 & 721934 & 721934 & 1513.8 & 0.000 \\
swath1 & 18164 & 59 & 14170 & 379.0712957 & 379.0712957 & 337.6 & 0.000 \\
swath2 & 41030 & 80 & 16642 & 385.1996929 & 385.1996929 & 663.2 & 0.000 \\
\hline Total (24) & 6740191 & 1845 & 166773 & & & 10927.8 & 0.000 \\
Geom. Mean & 46664.5 & & 2757.3 & & & 178.0 & \\
\hline & & & & & &
\end{tabular}

Table 15: dynamic reliability branching $\left(\eta_{\mathrm{rel}}=4-32, \lambda=4\right)$ 


\begin{tabular}{lrrrrrrr}
\hline Example & B \& B & Cuts & StrBra & Dual Bound & Primal Bound & Time & Gap \% \\
\hline aflow30a & 170547 & 22 & 397 & 1158 & 1158 & 391.7 & 0.000 \\
cap6000 & 5076 & 3 & 92 & -2451377 & -2451377 & 37.8 & 0.000 \\
gesa2-o & 79841 & 171 & 1841 & 25779856.37 & 25779856.37 & 284.3 & 0.000 \\
mas74 & 5296806 & 0 & 88 & 11801.18573 & 11801.18573 & 3066.4 & 0.000 \\
mas76 & 496370 & 0 & 69 & 40005.05414 & 40005.05414 & 242.2 & 0.000 \\
misc07 & 41902 & 71 & 266 & 2810 & 2810 & 113.2 & 0.000 \\
pk1 & 367763 & 0 & 54 & 11 & 11 & 200.8 & 0.000 \\
pp08aCUTS & 651 & 494 & 60 & 7350 & 7350 & 4.4 & 0.000 \\
qiu & 16148 & 1 & 48 & -132.873137 & -132.873137 & 371.2 & 0.000 \\
rout & 39192 & 68 & 426 & 1077.56 & 1077.56 & 122.2 & 0.000 \\
vpm2 & 17409 & 214 & 141 & 13.75 & 13.75 & 25.0 & 0.000 \\
ran8x32 & 31697 & 93 & 667 & 5247 & 5247 & 63.7 & 0.000 \\
ran10x26 & 68237 & 56 & 586 & 4270 & 4270 & 104.7 & 0.000 \\
ran12x21 & 159383 & 62 & 940 & 3664 & 3664 & 250.8 & 0.000 \\
ran13x13 & 97195 & 78 & 311 & 3252 & 3252 & 144.8 & 0.000 \\
mas284 & 21217 & 0 & 85 & 91405.72368 & 91405.72368 & 23.7 & 0.000 \\
prod1 & 65679 & 163 & 158 & -56 & -56 & 96.2 & 0.000 \\
bc1 & 35132 & 0 & 3189 & 3.338362548 & 3.338362548 & 1295.2 & 0.000 \\
bienst1 & 9248 & 23 & 27 & 46.75 & 46.75 & 83.3 & 0.000 \\
neos2 & 83146 & 29 & 13311 & 454.864697 & 454.864697 & 385.5 & 0.000 \\
neos3 & 736396 & 42 & 19218 & 323.1427543 & 378.749656 & 3600.0 & 17.208 \\
neos7 & 498573 & 112 & 5944 & 721934 & 721934 & 2377.6 & 0.000 \\
swath1 & 66995 & 59 & 2088 & 379.0712957 & 379.0712957 & 604.6 & 0.000 \\
swath2 & 258934 & 80 & 3551 & 385.1996929 & 385.1996929 & 2864.5 & 0.000 \\
\hline Total (24) & 8663537 & 1841 & 53557 & & & 16753.8 & 17.208 \\
Geom. Mean & 69501.0 & & 429.0 & & & 217.2 & \\
\hline & & & & & & &
\end{tabular}

Table 16: reliability branching $\left(\eta_{\text {rel }}=1, \lambda=8\right)$ 


\begin{tabular}{lrrrrrrr}
\hline Example & B \& B & Cuts & StrBra & Dual Bound & Primal Bound & Time & Gap \% \\
\hline aflow30a & 231706 & 22 & 1467 & 1158 & 1158 & 452.6 & 0.000 \\
cap6000 & 4474 & 3 & 331 & -2451377 & -2451377 & 37.1 & 0.000 \\
gesa2-o & 53054 & 171 & 1287 & 25779856.37 & 25779856.37 & 189.8 & 0.000 \\
mas74 & 5771953 & 0 & 341 & 11801.18573 & 11801.18573 & 3173.7 & 0.000 \\
mas76 & 427671 & 0 & 267 & 40005.05414 & 40005.05414 & 188.4 & 0.000 \\
misc07 & 42609 & 71 & 1394 & 2810 & 2810 & 117.1 & 0.000 \\
pk1 & 318561 & 0 & 204 & 11 & 11 & 186.6 & 0.000 \\
pp08aCUTS & 421 & 494 & 260 & 7350 & 7350 & 3.9 & 0.000 \\
qiu & 21411 & 1 & 188 & -132.8731565 & -132.8731565 & 445.4 & 0.000 \\
rout & 28642 & 68 & 1853 & 1077.56 & 1077.56 & 90.8 & 0.000 \\
vpm2 & 10494 & 214 & 544 & 13.75 & 13.75 & 15.4 & 0.000 \\
ran8x32 & 27887 & 93 & 2164 & 5247 & 5247 & 58.1 & 0.000 \\
ran10x26 & 51712 & 56 & 1612 & 4270 & 4270 & 87.5 & 0.000 \\
ran12x21 & 127131 & 62 & 1243 & 3664 & 3664 & 203.2 & 0.000 \\
ran13x13 & 101209 & 78 & 925 & 3252 & 3252 & 147.0 & 0.000 \\
mas284 & 20266 & 0 & 271 & 91405.72368 & 91405.72368 & 24.2 & 0.000 \\
prod1 & 58136 & 163 & 624 & -56 & -56 & 89.9 & 0.000 \\
bc1 & 28159 & 0 & 4921 & 3.338362548 & 3.338362548 & 1139.7 & 0.000 \\
bienst1 & 10303 & 23 & 104 & 46.75 & 46.75 & 104.9 & 0.000 \\
neos2 & 50790 & 29 & 13680 & 454.864697 & 454.864697 & 292.5 & 0.000 \\
neos3 & 347769 & 42 & 26934 & 368.842751 & 368.842751 & 1712.9 & 0.000 \\
neos7 & 477884 & 112 & 5148 & 721934 & 721934 & 2345.2 & 0.000 \\
swath1 & 20170 & 59 & 4174 & 379.0712957 & 379.0712957 & 218.3 & 0.000 \\
swath2 & 105974 & 80 & 4970 & 385.1996929 & 385.1996929 & 1173.0 & 0.000 \\
\hline Total (24) & 8338386 & 1841 & 74906 & & & 12497.2 & 0.000 \\
Geom. Mean & 54937.7 & & 1104.6 & & & 179.2 & \\
\hline & & & & & &
\end{tabular}

Table 17: reliability branching $\left(\eta_{\text {rel }}=4, \lambda=8\right)$ 


\begin{tabular}{lrrrrrrr}
\hline Example & B \& B & Cuts & StrBra & Dual Bound & Primal Bound & Time & Gap \% \\
\hline aflow30a & 171909 & 22 & 2865 & 1158 & 1158 & 368.2 & 0.000 \\
cap6000 & 4253 & 3 & 597 & -2451377 & -2451377 & 36.3 & 0.000 \\
gesa2-o & 50078 & 171 & 2178 & 25779856.37 & 25779856.37 & 195.7 & 0.000 \\
mas74 & 5425001 & 0 & 673 & 11801.18573 & 11801.18573 & 3104.2 & 0.000 \\
mas76 & 336826 & 0 & 558 & 40005.05414 & 40005.05414 & 161.2 & 0.000 \\
misc07 & 55740 & 71 & 3262 & 2810 & 2810 & 150.2 & 0.000 \\
pk1 & 311611 & 0 & 402 & 11 & 11 & 170.3 & 0.000 \\
pp08aCUTS & 464 & 494 & 438 & 7350 & 7350 & 5.5 & 0.000 \\
qiu & 14847 & 1 & 370 & -132.8731516 & -132.8731516 & 341.9 & 0.000 \\
rout & 19743 & 68 & 3473 & 1077.56 & 1077.56 & 81.3 & 0.000 \\
vpm2 & 10054 & 214 & 1089 & 13.75 & 13.75 & 16.3 & 0.000 \\
ran8x32 & 21092 & 93 & 2534 & 5247 & 5247 & 47.2 & 0.000 \\
ran10x26 & 48626 & 56 & 3417 & 4270 & 4270 & 82.0 & 0.000 \\
ran12x21 & 124455 & 62 & 2591 & 3664 & 3664 & 199.0 & 0.000 \\
ran13x13 & 93939 & 78 & 1521 & 3252 & 3252 & 136.1 & 0.000 \\
mas284 & 20360 & 0 & 477 & 91405.72368 & 91405.72368 & 24.1 & 0.000 \\
prod1 & 62689 & 163 & 1173 & -56 & -56 & 97.8 & 0.000 \\
bc1 & 25196 & 0 & 5092 & 3.338362548 & 3.338362548 & 1153.0 & 0.000 \\
bienst1 & 9951 & 23 & 206 & 46.75 & 46.75 & 91.0 & 0.000 \\
neos2 & 30790 & 29 & 13994 & 454.864697 & 454.864697 & 222.2 & 0.000 \\
neos3 & 626894 & 42 & 54459 & 368.842751 & 368.842751 & 3145.8 & 0.000 \\
neos7 & 252766 & 112 & 17062 & 721934 & 721934 & 1350.3 & 0.000 \\
swath1 & 10615 & 59 & 6474 & 379.0712957 & 379.0712957 & 166.2 & 0.000 \\
swath2 & 85510 & 80 & 8640 & 385.1996929 & 385.1996929 & 1034.6 & 0.000 \\
\hline Total (24) & 7813409 & 1841 & 133545 & & & 12380.2 & 0.000 \\
Geom. Mean & 48377.3 & & 1998.0 & & & 170.2 & \\
\hline & & & & & &
\end{tabular}

Table 18: reliability branching $\left(\eta_{\text {rel }}=8, \lambda=8\right)$ 


\begin{tabular}{lrrrrrrr}
\hline Example & B \& B & Cuts & StrBra & Dual Bound & Primal Bound & Time & Gap \% \\
\hline aflow30a & 163331 & 22 & 5690 & 1158 & 1158 & 364.2 & 0.000 \\
cap6000 & 4998 & 3 & 1099 & -2451377 & -2451377 & 49.1 & 0.000 \\
gesa2-o & 39751 & 171 & 3874 & 25779856.37 & 25779856.37 & 160.6 & 0.000 \\
mas74 & 5387375 & 0 & 1316 & 11801.18573 & 11801.18573 & 3068.4 & 0.000 \\
mas76 & 304694 & 0 & 1029 & 40005.05414 & 40005.05414 & 136.1 & 0.000 \\
misc07 & 52422 & 71 & 5859 & 2810 & 2810 & 144.7 & 0.000 \\
pk1 & 323364 & 0 & 793 & 11 & 11 & 179.6 & 0.000 \\
pp08aCUTS & 397 & 494 & 909 & 7350 & 7350 & 7.7 & 0.000 \\
qiu & 12550 & 1 & 736 & -132.8731399 & -132.8731399 & 332.8 & 0.000 \\
rout & 19531 & 68 & 5356 & 1077.56 & 1077.56 & 93.2 & 0.000 \\
vpm2 & 10714 & 214 & 2040 & 13.75 & 13.75 & 18.6 & 0.000 \\
ran8x32 & 22464 & 93 & 5366 & 5247 & 5247 & 54.2 & 0.000 \\
ran10x26 & 33990 & 56 & 8612 & 4270 & 4270 & 74.5 & 0.000 \\
ran12x21 & 129279 & 62 & 4940 & 3664 & 3664 & 216.4 & 0.000 \\
ran13x13 & 84623 & 78 & 3222 & 3252 & 3252 & 116.5 & 0.000 \\
mas284 & 19818 & 0 & 995 & 91405.72368 & 91405.72368 & 26.2 & 0.000 \\
prod1 & 60253 & 163 & 2310 & -56 & -56 & 97.2 & 0.000 \\
bc1 & 23936 & 0 & 7496 & 3.338362548 & 3.338362548 & 1203.7 & 0.000 \\
bienst1 & 12210 & 23 & 398 & 46.75 & 46.75 & 106.7 & 0.000 \\
neos2 & 16624 & 29 & 16704 & 454.864697 & 454.864697 & 187.8 & 0.000 \\
neos3 & 435159 & 42 & 42205 & 368.842751 & 368.842751 & 2208.7 & 0.000 \\
neos7 & 390198 & 112 & 41645 & 721934 & 721934 & 2508.0 & 0.000 \\
swath1 & 13339 & 59 & 11324 & 379.0712957 & 379.0712957 & 241.1 & 0.000 \\
swath2 & 18380 & 80 & 11218 & 385.1996929 & 385.1996929 & 350.9 & 0.000 \\
\hline Total (24) & 7579400 & 1841 & 185136 & & & 11946.7 & 0.000 \\
Geom. Mean & 43311.9 & & 3589.7 & & & 171.3 & \\
\hline & & & & & & &
\end{tabular}

Table 19: reliability branching $\left(\eta_{\text {rel }}=16, \lambda=8\right)$ 


\begin{tabular}{lrrrrrrr}
\hline Example & B \& B & Cuts & StrBra & Dual Bound & Primal Bound & Time & Gap \% \\
\hline aflow30a & 145980 & 22 & 11147 & 1158 & 1158 & 305.3 & 0.000 \\
cap6000 & 4010 & 3 & 1695 & -2451377 & -2451377 & 42.1 & 0.000 \\
gesa2-o & 40222 & 171 & 6292 & 25779856.37 & 25779856.37 & 168.6 & 0.000 \\
mas74 & 4999610 & 0 & 2480 & 11801.18573 & 11801.18573 & 2823.9 & 0.000 \\
mas76 & 454361 & 0 & 2234 & 40005.05414 & 40005.05414 & 205.6 & 0.000 \\
misc07 & 48338 & 71 & 11508 & 2810 & 2810 & 155.7 & 0.000 \\
pk1 & 352716 & 0 & 1551 & 11 & 11 & 204.2 & 0.000 \\
pp08aCUTS & 317 & 494 & 1599 & 7350 & 7350 & 10.8 & 0.000 \\
qiu & 12777 & 1 & 1476 & -132.8731595 & -132.8731595 & 366.7 & 0.000 \\
rout & 20773 & 68 & 11017 & 1077.56 & 1077.56 & 122.6 & 0.000 \\
vpm2 & 8698 & 214 & 3315 & 13.75 & 13.75 & 18.8 & 0.000 \\
ran8x32 & 18155 & 93 & 6352 & 5247 & 5247 & 51.6 & 0.000 \\
ran10x26 & 39713 & 56 & 8177 & 4270 & 4270 & 91.7 & 0.000 \\
ran12x21 & 114304 & 62 & 9862 & 3664 & 3664 & 207.0 & 0.000 \\
ran13x13 & 84047 & 78 & 6529 & 3252 & 3252 & 135.8 & 0.000 \\
mas284 & 18623 & 0 & 1773 & 91405.72368 & 91405.72368 & 24.9 & 0.000 \\
prod1 & 59335 & 163 & 4284 & -56 & -56 & 103.6 & 0.000 \\
bc1 & 18385 & 0 & 10011 & 3.338362548 & 3.338362548 & 1213.1 & 0.000 \\
bienst1 & 10229 & 23 & 736 & 46.75 & 46.75 & 90.6 & 0.000 \\
neos2 & 9823 & 29 & 20941 & 454.864697 & 454.864697 & 177.6 & 0.000 \\
neos3 & 430739 & 42 & 65191 & 368.842751 & 368.842751 & 2559.9 & 0.000 \\
neos7 & 256009 & 112 & 29359 & 721934 & 721934 & 1621.4 & 0.000 \\
swath1 & 25453 & 59 & 20225 & 379.0712957 & 379.0712957 & 456.0 & 0.000 \\
swath2 & 35219 & 80 & 21728 & 385.1996929 & 385.1996929 & 678.2 & 0.000 \\
\hline Total (24) & 7207836 & 1841 & 259482 & & & 11835.7 & 0.000 \\
Geom. Mean & 42047.5 & & 5913.2 & & & 186.5 & \\
\hline & & & & & &
\end{tabular}

Table 20: reliability branching $\left(\eta_{\text {rel }}=32, \lambda=8\right)$ 


\begin{tabular}{lrrrrrrr}
\hline Example & B \& B & Cuts & StrBra & Dual Bound & Primal Bound & Time & Gap \% \\
\hline aflow30a & 147325 & 22 & 3236 & 1158 & 1158 & 290.9 & 0.000 \\
cap6000 & 4474 & 3 & 331 & -2451377 & -2451377 & 35.5 & 0.000 \\
gesa2-o & 49412 & 171 & 1597 & 25779856.37 & 25779856.37 & 187.3 & 0.000 \\
mas74 & 5328123 & 0 & 709 & 11801.18573 & 11801.18573 & 3077.8 & 0.000 \\
mas76 & 374046 & 0 & 545 & 40005.05414 & 40005.05414 & 171.1 & 0.000 \\
misc07 & 58624 & 71 & 1474 & 2810 & 2810 & 160.4 & 0.000 \\
pk1 & 365257 & 0 & 491 & 11 & 11 & 214.8 & 0.000 \\
pp08aCUTS & 541 & 494 & 521 & 7350 & 7350 & 6.4 & 0.000 \\
qiu & 15641 & 1 & 365 & -132.8731601 & -132.8731601 & 370.1 & 0.000 \\
rout & 84775 & 68 & 3820 & 1077.56 & 1077.56 & 236.2 & 0.000 \\
vpm2 & 9272 & 214 & 1100 & 13.75 & 13.75 & 15.3 & 0.000 \\
ran8x32 & 18916 & 93 & 2772 & 5247 & 5247 & 44.3 & 0.000 \\
ran10x26 & 47409 & 56 & 2381 & 4270 & 4270 & 82.9 & 0.000 \\
ran12x21 & 118905 & 62 & 2678 & 3664 & 3664 & 189.0 & 0.000 \\
ran13x13 & 90486 & 78 & 1898 & 3252 & 3252 & 132.4 & 0.000 \\
mas284 & 21470 & 0 & 405 & 91405.72368 & 91405.72368 & 28.1 & 0.000 \\
prod1 & 61400 & 163 & 686 & -56 & -56 & 97.2 & 0.000 \\
bc1 & 28159 & 0 & 4921 & 3.338362548 & 3.338362548 & 1154.7 & 0.000 \\
bienst1 & 11446 & 23 & 271 & 46.75 & 46.75 & 124.2 & 0.000 \\
neos2 & 39797 & 29 & 17509 & 454.864697 & 454.864697 & 315.9 & 0.000 \\
neos3 & 676114 & 42 & 43070 & 368.842751 & 368.842751 & 2918.1 & 0.000 \\
neos7 & 317136 & 112 & 18765 & 721934 & 721934 & 1619.1 & 0.000 \\
swath1 & 12704 & 59 & 7784 & 379.0712957 & 379.0712957 & 204.5 & 0.000 \\
swath2 & 22656 & 80 & 7533 & 385.1996929 & 385.1996929 & 347.3 & 0.000 \\
\hline Total (24) & 7904088 & 1841 & 124862 & & & 12023.3 & 0.000 \\
Geom. Mean & 50937.3 & & 1882.9 & & & 178.9 & \\
\hline & & & & & &
\end{tabular}

Table 21: dynamic reliability branching $\left(\eta_{\text {rel }}=4-16, \lambda=8\right)$ 


\begin{tabular}{lrrrrrrr}
\hline Example & B \& B & Cuts & StrBra & Dual Bound & Primal Bound & Time & Gap \% \\
\hline aflow30a & 162318 & 22 & 6400 & 1158 & 1158 & 389.1 & 0.000 \\
cap6000 & 4552 & 3 & 326 & -2451377 & -2451377 & 35.6 & 0.000 \\
gesa2-o & 45052 & 171 & 2292 & 25779856.37 & 25779856.37 & 174.2 & 0.000 \\
mas74 & 5519583 & 0 & 1181 & 11801.18573 & 11801.18573 & 3084.8 & 0.000 \\
mas76 & 382316 & 0 & 804 & 40005.05414 & 40005.05414 & 174.5 & 0.000 \\
misc07 & 48927 & 71 & 2664 & 2810 & 2810 & 137.5 & 0.000 \\
pk1 & 330389 & 0 & 861 & 11 & 11 & 172.3 & 0.000 \\
pp08aCUTS & 385 & 494 & 852 & 7350 & 7350 & 7.1 & 0.000 \\
qiu & 12348 & 1 & 602 & -132.8731458 & -132.8731458 & 306.5 & 0.000 \\
rout & 25409 & 68 & 6910 & 1077.56 & 1077.56 & 123.2 & 0.000 \\
vpm2 & 10858 & 214 & 1626 & 13.75 & 13.75 & 18.3 & 0.000 \\
ran8x32 & 23086 & 93 & 4176 & 5247 & 5247 & 57.1 & 0.000 \\
ran10x26 & 44861 & 56 & 4301 & 4270 & 4270 & 87.0 & 0.000 \\
ran12x21 & 110413 & 62 & 4515 & 3664 & 3664 & 195.6 & 0.000 \\
ran13x13 & 82666 & 78 & 3051 & 3252 & 3252 & 125.8 & 0.000 \\
mas284 & 18756 & 0 & 577 & 91405.72368 & 91405.72368 & 23.7 & 0.000 \\
prod1 & 60658 & 163 & 773 & -56 & -56 & 95.5 & 0.000 \\
bc1 & 28159 & 0 & 4921 & 3.338362548 & 3.338362548 & 1195.4 & 0.000 \\
bienst1 & 12295 & 23 & 421 & 46.75 & 46.75 & 108.0 & 0.000 \\
neos2 & 25223 & 29 & 15719 & 454.864697 & 454.864697 & 177.9 & 0.000 \\
neos3 & 660840 & 42 & 41400 & 356.1420421 & 370.6295699 & 3600.0 & 4.068 \\
neos7 & 235967 & 112 & 11209 & 721934 & 721934 & 1086.8 & 0.000 \\
swath1 & 11936 & 59 & 11453 & 379.0712957 & 379.0712957 & 235.1 & 0.000 \\
swath2 & 14473 & 80 & 11245 & 385.1996929 & 385.1996929 & 307.9 & 0.000 \\
\hline Total (24) & 7871470 & 1841 & 138279 & & & 11918.9 & 4.068 \\
Geom. Mean & 44566.0 & & 2637.9 & & & 168.4 & \\
\hline & & & & & &
\end{tabular}

Table 22: dynamic reliability branching $\left(\eta_{\text {rel }}=4-32, \lambda=8\right)$ 


\begin{tabular}{lrrrrrrr}
\hline Example & B \& B & Cuts & StrBra & Dual Bound & Primal Bound & Time & Gap \% \\
\hline aflow30a & 225745 & 22 & 13683 & 1158 & 1158 & 488.8 & 0.000 \\
cap6000 & 4345 & 3 & 355 & -2451377 & -2451377 & 33.5 & 0.000 \\
gesa2-o & 42066 & 171 & 3426 & 25779856.37 & 25779856.37 & 168.1 & 0.000 \\
mas74 & 5512555 & 0 & 2145 & 11801.18573 & 11801.18573 & 3346.6 & 0.000 \\
mas76 & 498782 & 0 & 1558 & 40005.05414 & 40005.05414 & 238.2 & 0.000 \\
misc07 & 39334 & 71 & 4535 & 2810 & 2810 & 121.4 & 0.000 \\
pk1 & 357602 & 0 & 1381 & 11 & 11 & 195.4 & 0.000 \\
pp08aCUTS & 365 & 494 & 1084 & 7350 & 7350 & 8.8 & 0.000 \\
qiu & 12767 & 1 & 975 & -132.8731517 & -132.8731517 & 339.1 & 0.000 \\
rout & 26396 & 68 & 11477 & 1077.56 & 1077.56 & 159.1 & 0.000 \\
vpm2 & 10531 & 214 & 2762 & 13.75 & 13.75 & 21.2 & 0.000 \\
ran8x32 & 20511 & 93 & 7465 & 5247 & 5247 & 59.5 & 0.000 \\
ran10x26 & 41057 & 56 & 8448 & 4270 & 4270 & 94.9 & 0.000 \\
ran12x21 & 120191 & 62 & 8681 & 3664 & 3664 & 213.3 & 0.000 \\
ran13x13 & 78051 & 78 & 6255 & 3252 & 3252 & 116.1 & 0.000 \\
mas284 & 21132 & 0 & 897 & 91405.72368 & 91405.72368 & 26.9 & 0.000 \\
prod1 & 65618 & 163 & 940 & -56 & -56 & 100.7 & 0.000 \\
bc1 & 28159 & 0 & 4921 & 3.338362548 & 3.338362548 & 1128.2 & 0.000 \\
bienst1 & 10173 & 23 & 633 & 46.75 & 46.75 & 105.8 & 0.000 \\
neos2 & 60902 & 29 & 19507 & 454.864697 & 454.864697 & 338.4 & 0.000 \\
neos3 & 552853 & 42 & 45623 & 368.842751 & 368.842751 & 3020.7 & 0.000 \\
neos7 & 278237 & 112 & 17159 & 721934 & 721934 & 1359.9 & 0.000 \\
swath1 & 11209 & 59 & 16460 & 379.0712957 & 379.0712957 & 277.1 & 0.000 \\
swath2 & 21260 & 80 & 20233 & 385.1996929 & 385.1996929 & 491.2 & 0.000 \\
\hline Total (24) & 8039841 & 1841 & 200603 & & & 12452.9 & 0.000 \\
Geom. Mean & 47137.5 & & 4108.1 & & & 189.3 & \\
\hline & & & & & &
\end{tabular}

Table 23: dynamic reliability branching $\left(\eta_{\text {rel }}=4-64, \lambda=8\right)$ 


\begin{tabular}{lrrrrrrr}
\hline Example & B \& B & Cuts & StrBra & Dual Bound & Primal Bound & Time & Gap \% \\
\hline aflow30a & 188650 & 22 & 9662 & 1158 & 1158 & 425.4 & 0.000 \\
cap6000 & 4474 & 3 & 331 & -2451377 & -2451377 & 35.6 & 0.000 \\
gesa2-o & 48071 & 171 & 2604 & 25779856.37 & 25779856.37 & 184.6 & 0.000 \\
mas74 & 5062536 & 0 & 6405 & 11801.18573 & 11801.18573 & 2904.2 & 0.000 \\
mas76 & 498594 & 0 & 5312 & 40005.05414 & 40005.05414 & 229.1 & 0.000 \\
misc07 & 56391 & 71 & 2755 & 2810 & 2810 & 147.9 & 0.000 \\
pk1 & 386217 & 0 & 4443 & 11 & 11 & 234.3 & 0.000 \\
pp08aCUTS & 225 & 494 & 2067 & 7350 & 7350 & 13.0 & 0.000 \\
qiu & 18001 & 1 & 369 & -132.8731399 & -132.8731399 & 430.9 & 0.000 \\
rout & 13521 & 68 & 16178 & 1077.56 & 1077.56 & 125.1 & 0.000 \\
vpm2 & 6664 & 214 & 6588 & 13.75 & 13.75 & 22.9 & 0.000 \\
ran8x32 & 16388 & 93 & 11606 & 5247 & 5247 & 59.6 & 0.000 \\
ran10x26 & 42209 & 56 & 11470 & 4270 & 4270 & 92.9 & 0.000 \\
ran12x21 & 113008 & 62 & 12514 & 3664 & 3664 & 209.9 & 0.000 \\
ran13x13 & 76934 & 78 & 9969 & 3252 & 3252 & 124.3 & 0.000 \\
mas284 & 19827 & 0 & 386 & 91405.72368 & 91405.72368 & 24.6 & 0.000 \\
prod1 & 62556 & 163 & 2997 & -56 & -56 & 103.9 & 0.000 \\
bc1 & 28159 & 0 & 4921 & 3.338362548 & 3.338362548 & 1133.8 & 0.000 \\
bienst1 & 11118 & 23 & 1148 & 46.75 & 46.75 & 99.0 & 0.000 \\
neos2 & 37892 & 29 & 11182 & 454.864697 & 454.864697 & 209.3 & 0.000 \\
neos3 & 347769 & 42 & 26934 & 368.842751 & 368.842751 & 1708.5 & 0.000 \\
neos7 & 477884 & 112 & 5148 & 721934 & 721934 & 2299.4 & 0.000 \\
swath1 & 20170 & 59 & 4174 & 379.0712957 & 379.0712957 & 218.9 & 0.000 \\
swath2 & 105974 & 80 & 4970 & 385.1996929 & 385.1996929 & 1165.3 & 0.000 \\
\hline Total (24) & 7643232 & 1841 & 164133 & & & 12202.3 & 0.000 \\
Geom. Mean & 48268.9 & & 4214.2 & & & 194.9 & \\
\hline & & & & & &
\end{tabular}

Table 24: dynamic reliability branching $\left(\eta_{\text {rel }}=4-128, \lambda=8\right)$ 


\begin{tabular}{lrrrrrrr}
\hline Example & B \& B & Cuts & StrBra & Dual Bound & Primal Bound & Time & Gap \% \\
\hline aflow30a & 184984 & 22 & 407 & 1158 & 1158 & 414.9 & 0.000 \\
cap6000 & 5076 & 3 & 92 & -2451377 & -2451377 & 37.6 & 0.000 \\
gesa2-o & 66583 & 171 & 627 & 25779856.37 & 25779856.37 & 248.3 & 0.000 \\
mas74 & 4207847 & 0 & 96 & 11801.18573 & 11801.18573 & 2020.7 & 0.000 \\
mas76 & 496370 & 0 & 69 & 40005.05414 & 40005.05414 & 239.5 & 0.000 \\
misc07 & 41535 & 71 & 387 & 2810 & 2810 & 119.6 & 0.000 \\
pk1 & 367763 & 0 & 54 & 11 & 11 & 193.7 & 0.000 \\
pp08aCUTS & 651 & 494 & 60 & 7350 & 7350 & 4.5 & 0.000 \\
qiu & 16148 & 1 & 48 & -132.873137 & -132.873137 & 367.7 & 0.000 \\
rout & 45318 & 68 & 821 & 1077.56 & 1077.56 & 121.6 & 0.000 \\
vpm2 & 13124 & 214 & 122 & 13.75 & 13.75 & 17.8 & 0.000 \\
ran8x32 & 46039 & 93 & 1577 & 5247 & 5247 & 83.8 & 0.000 \\
ran10x26 & 63050 & 56 & 692 & 4270 & 4270 & 94.7 & 0.000 \\
ran12x21 & 208719 & 62 & 633 & 3664 & 3664 & 353.9 & 0.000 \\
ran13x13 & 115793 & 78 & 187 & 3252 & 3252 & 166.5 & 0.000 \\
mas284 & 21964 & 0 & 106 & 91405.72368 & 91405.72368 & 25.2 & 0.000 \\
prod1 & 64193 & 156 & 153 & -56 & -56 & 98.0 & 0.000 \\
bc1 & 35132 & 0 & 3189 & 3.338362548 & 3.338362548 & 1285.6 & 0.000 \\
bienst1 & 8703 & 23 & 28 & 46.75 & 46.75 & 81.6 & 0.000 \\
neos2 & 178106 & 29 & 12153 & 454.864697 & 454.864697 & 645.5 & 0.000 \\
neos3 & 817180 & 42 & 19520 & 363.852925 & 368.842751 & 3600.0 & 1.371 \\
neos7 & 417083 & 112 & 1502 & 721934 & 721934 & 2121.0 & 0.000 \\
swath1 & 66995 & 59 & 2088 & 379.0712957 & 379.0712957 & 609.9 & 0.000 \\
swath2 & 258934 & 80 & 3551 & 385.1996929 & 385.1996929 & 2874.2 & 0.000 \\
\hline Total (24) & 7747290 & 1834 & 48162 & & & 15825.8 & 1.371 \\
Geom. Mean & 72159.1 & & 408.4 & & & 220.0 & \\
\hline & & & & & & &
\end{tabular}

Table 25: reliability branching $\left(\eta_{\text {rel }}=1, \lambda=\infty\right)$ 


\begin{tabular}{lrrrrrrr}
\hline Example & B \& B & Cuts & StrBra & Dual Bound & Primal Bound & Time & Gap \% \\
\hline aflow30a & 128910 & 22 & 1463 & 1158 & 1158 & 296.7 & 0.000 \\
cap6000 & 4474 & 3 & 331 & -2451377 & -2451377 & 35.1 & 0.000 \\
gesa2-o & 48818 & 171 & 1354 & 25779856.37 & 25779856.37 & 184.8 & 0.000 \\
mas74 & 6290002 & 0 & 374 & 11794.87806 & 11801.18573 & 3600.0 & 0.053 \\
mas76 & 459034 & 0 & 286 & 40005.05414 & 40005.05414 & 214.4 & 0.000 \\
misc07 & 54790 & 71 & 1053 & 2810 & 2810 & 145.2 & 0.000 \\
pk1 & 328700 & 0 & 205 & 11 & 11 & 187.4 & 0.000 \\
pp08aCUTS & 570 & 494 & 230 & 7350 & 7350 & 4.9 & 0.000 \\
qiu & 22147 & 1 & 188 & -132.8731517 & -132.8731517 & 478.6 & 0.000 \\
rout & 37624 & 68 & 1803 & 1077.56 & 1077.56 & 149.9 & 0.000 \\
vpm2 & 13934 & 214 & 604 & 13.75 & 13.75 & 20.3 & 0.000 \\
ran8x32 & 29101 & 93 & 2645 & 5247 & 5247 & 64.9 & 0.000 \\
ran10x26 & 51788 & 56 & 1511 & 4270 & 4270 & 82.2 & 0.000 \\
ran12x21 & 130173 & 62 & 1413 & 3664 & 3664 & 218.6 & 0.000 \\
ran13x13 & 89596 & 78 & 591 & 3252 & 3252 & 126.4 & 0.000 \\
mas284 & 22051 & 0 & 264 & 91405.72368 & 91405.72368 & 26.5 & 0.000 \\
prod1 & 69166 & 156 & 525 & -56 & -56 & 108.1 & 0.000 \\
bc1 & 28159 & 0 & 4921 & 3.338362548 & 3.338362548 & 1123.8 & 0.000 \\
bienst1 & 13752 & 23 & 108 & 46.75 & 46.75 & 140.2 & 0.000 \\
neos2 & 38746 & 29 & 9972 & 454.864697 & 454.864697 & 221.9 & 0.000 \\
neos3 & 718479 & 42 & 32418 & 354.3425985 & 369.4101772 & 3600.0 & 4.252 \\
neos7 & 357385 & 112 & 5643 & 721934 & 721934 & 1690.7 & 0.000 \\
swath1 & 29728 & 59 & 5713 & 379.0712957 & 379.0712957 & 318.2 & 0.000 \\
swath2 & 101596 & 80 & 5010 & 385.1996929 & 385.1996929 & 1219.4 & 0.000 \\
\hline Total (24) & 9068723 & 1834 & 78625 & & & 14258.1 & 4.306 \\
Geom. Mean & 58886.4 & & 1096.6 & & & 195.8 & \\
\hline & & & & & & &
\end{tabular}

Table 26: reliability branching $\left(\eta_{\text {rel }}=4, \lambda=\infty\right)$ 


\begin{tabular}{lrrrrrrr}
\hline Example & B \& B & Cuts & StrBra & Dual Bound & Primal Bound & Time & Gap \% \\
\hline aflow30a & 144418 & 22 & 2880 & 1158 & 1158 & 315.6 & 0.000 \\
cap6000 & 4253 & 3 & 597 & -2451377 & -2451377 & 36.1 & 0.000 \\
gesa2-o & 51454 & 171 & 2435 & 25779856.37 & 25779856.37 & 211.3 & 0.000 \\
mas74 & 6094785 & 0 & 675 & 11801.18573 & 11801.18573 & 3313.9 & 0.000 \\
mas76 & 380456 & 0 & 506 & 40005.05414 & 40005.05414 & 162.4 & 0.000 \\
misc07 & 55242 & 71 & 3064 & 2810 & 2810 & 152.9 & 0.000 \\
pk1 & 237780 & 0 & 399 & 11 & 11 & 119.2 & 0.000 \\
pp08aCUTS & 400 & 494 & 453 & 7350 & 7350 & 5.3 & 0.000 \\
qiu & 19736 & 1 & 373 & -132.8731462 & -132.8731462 & 456.5 & 0.000 \\
rout & 88970 & 68 & 3399 & 1077.56 & 1077.56 & 227.6 & 0.000 \\
vpm2 & 15286 & 214 & 1182 & 13.75 & 13.75 & 23.8 & 0.000 \\
ran8x32 & 28033 & 93 & 2823 & 5247 & 5247 & 60.8 & 0.000 \\
ran10x26 & 43295 & 56 & 4589 & 4270 & 4270 & 76.2 & 0.000 \\
ran12x21 & 129531 & 62 & 3020 & 3664 & 3664 & 213.3 & 0.000 \\
ran13x13 & 101314 & 78 & 1492 & 3252 & 3252 & 146.2 & 0.000 \\
mas284 & 22644 & 0 & 486 & 91405.72368 & 91405.72368 & 29.0 & 0.000 \\
prod1 & 62944 & 156 & 1092 & -56 & -56 & 103.5 & 0.000 \\
bc1 & 25196 & 0 & 5092 & 3.338362548 & 3.338362548 & 1113.2 & 0.000 \\
bienst1 & 11677 & 23 & 209 & 46.75 & 46.75 & 115.1 & 0.000 \\
neos2 & 26049 & 29 & 15229 & 454.864697 & 454.864697 & 193.4 & 0.000 \\
neos3 & 582896 & 42 & 57473 & 368.6645774 & 368.842751 & 3600.0 & 0.048 \\
neos7 & 359672 & 112 & 9819 & 721934 & 721934 & 1997.9 & 0.000 \\
swath1 & 32431 & 59 & 8610 & 379.0712957 & 379.0712957 & 384.5 & 0.000 \\
swath2 & 32583 & 80 & 9644 & 385.1996929 & 385.1996929 & 505.4 & 0.000 \\
\hline Total (24) & 8551045 & 1834 & 135541 & & & 13563.0 & 0.048 \\
Geom. Mean & 54118.3 & & 2042.9 & & & 189.6 & \\
\hline & & & & & & &
\end{tabular}

Table 27: reliability branching $\left(\eta_{\text {rel }}=8, \lambda=\infty\right)$ 


\begin{tabular}{lrrrrrrr}
\hline Example & B \& B & Cuts & StrBra & Dual Bound & Primal Bound & Time & Gap \% \\
\hline aflow30a & 144316 & 22 & 5506 & 1158 & 1158 & 344.2 & 0.000 \\
cap6000 & 4998 & 3 & 1099 & -2451377 & -2451377 & 46.6 & 0.000 \\
gesa2-o & 46488 & 171 & 3745 & 25779856.37 & 25779856.37 & 181.1 & 0.000 \\
mas74 & 3990024 & 0 & 1222 & 11801.18573 & 11801.18573 & 1954.9 & 0.000 \\
mas76 & 439700 & 0 & 997 & 40005.05414 & 40005.05414 & 201.7 & 0.000 \\
misc07 & 53696 & 71 & 5570 & 2810 & 2810 & 150.0 & 0.000 \\
pk1 & 366697 & 0 & 776 & 11 & 11 & 219.8 & 0.000 \\
pp08aCUTS & 355 & 494 & 878 & 7350 & 7350 & 7.2 & 0.000 \\
qiu & 33771 & 1 & 738 & -132.873154 & -132.873154 & 697.6 & 0.000 \\
rout & 21702 & 68 & 6949 & 1077.56 & 1077.56 & 109.5 & 0.000 \\
vpm2 & 12972 & 214 & 2561 & 13.75 & 13.75 & 22.4 & 0.000 \\
ran8x32 & 18647 & 93 & 4109 & 5247 & 5247 & 47.4 & 0.000 \\
ran10x26 & 50813 & 56 & 4297 & 4270 & 4270 & 99.2 & 0.000 \\
ran12x21 & 115856 & 62 & 6644 & 3664 & 3664 & 204.8 & 0.000 \\
ran13x13 & 79323 & 78 & 3509 & 3252 & 3252 & 122.7 & 0.000 \\
mas284 & 20083 & 0 & 951 & 91405.72371 & 91405.72371 & 25.8 & 0.000 \\
prod1 & 60939 & 156 & 1987 & -56 & -56 & 95.8 & 0.000 \\
bc1 & 23936 & 0 & 7496 & 3.338362548 & 3.338362548 & 1201.2 & 0.000 \\
bienst1 & 9458 & 23 & 403 & 46.75 & 46.75 & 81.6 & 0.000 \\
neos2 & 24640 & 29 & 18651 & 454.864697 & 454.864697 & 233.4 & 0.000 \\
neos3 & 576820 & 42 & 54772 & 368.842751 & 368.842751 & 3080.0 & 0.000 \\
neos7 & 391371 & 112 & 35066 & 721934 & 721934 & 2437.4 & 0.000 \\
swath1 & 16303 & 59 & 11900 & 379.0712957 & 379.0712957 & 280.0 & 0.000 \\
swath2 & 64524 & 80 & 16394 & 385.1996929 & 385.1996929 & 922.0 & 0.000 \\
\hline Total (24) & 6567432 & 1834 & 196220 & & & 12766.4 & 0.000 \\
Geom. Mean & 49839.9 & & 3601.0 & & & 191.6 & \\
\hline & & & & & & &
\end{tabular}

Table 28: reliability branching $\left(\eta_{\text {rel }}=16, \lambda=\infty\right)$ 


\begin{tabular}{lrrrrrrr}
\hline Example & B \& B & Cuts & StrBra & Dual Bound & Primal Bound & Time & Gap \% \\
\hline aflow30a & 151594 & 22 & 11085 & 1158 & 1158 & 367.8 & 0.000 \\
cap6000 & 4010 & 3 & 1695 & -2451377 & -2451377 & 42.1 & 0.000 \\
gesa2-o & 37936 & 171 & 6668 & 25779856.37 & 25779856.37 & 168.4 & 0.000 \\
mas74 & 5365880 & 0 & 2437 & 11801.18573 & 11801.18573 & 3006.0 & 0.000 \\
mas76 & 452126 & 0 & 2002 & 40005.05414 & 40005.05414 & 217.7 & 0.000 \\
misc07 & 53100 & 71 & 11269 & 2810 & 2810 & 172.0 & 0.000 \\
pk1 & 298672 & 0 & 1560 & 11 & 11 & 165.7 & 0.000 \\
pp08aCUTS & 227 & 494 & 1413 & 7350 & 7350 & 9.6 & 0.000 \\
qiu & 25413 & 1 & 1476 & -132.873155 & -132.873155 & 661.3 & 0.000 \\
rout & 16069 & 68 & 9722 & 1077.56 & 1077.56 & 112.8 & 0.000 \\
vpm2 & 10261 & 214 & 3324 & 13.75 & 13.75 & 20.9 & 0.000 \\
ran8x32 & 12774 & 93 & 6518 & 5247 & 5247 & 41.9 & 0.000 \\
ran10x26 & 37628 & 56 & 10732 & 4270 & 4270 & 88.4 & 0.000 \\
ran12x21 & 109430 & 62 & 10258 & 3664 & 3664 & 200.9 & 0.000 \\
ran13x13 & 86213 & 78 & 5551 & 3252 & 3252 & 145.6 & 0.000 \\
mas284 & 19414 & 0 & 1775 & 91405.72368 & 91405.72368 & 25.4 & 0.000 \\
prod1 & 63365 & 156 & 3836 & -56 & -56 & 105.7 & 0.000 \\
bc1 & 18385 & 0 & 10011 & 3.338362548 & 3.338362548 & 1148.8 & 0.000 \\
bienst1 & 9377 & 23 & 740 & 46.75 & 46.75 & 89.6 & 0.000 \\
neos2 & 7598 & 29 & 21802 & 454.864697 & 454.864697 & 181.5 & 0.000 \\
neos3 & 289503 & 42 & 73905 & 368.842751 & 368.842751 & 1893.4 & 0.000 \\
neos7 & 357720 & 112 & 37595 & 721934 & 721934 & 2146.8 & 0.000 \\
swath1 & 20805 & 59 & 22796 & 379.0712957 & 379.0712957 & 448.6 & 0.000 \\
swath2 & 55442 & 80 & 23652 & 385.1996929 & 385.1996929 & 933.2 & 0.000 \\
\hline Total (24) & 7502942 & 1834 & 281822 & & & 12393.6 & 0.000 \\
Geom. Mean & 41636.1 & & 6000.7 & & & 192.5 & \\
\hline & & & & & & &
\end{tabular}

Table 29: reliability branching $\left(\eta_{\text {rel }}=32, \lambda=\infty\right)$ 


\begin{tabular}{lrrrrrrr}
\hline Example & B \& B & Cuts & StrBra & Dual Bound & Primal Bound & Time & Gap \% \\
\hline aflow30a & 198089 & 22 & 247 & 1158 & 1158 & 380.1 & 0.000 \\
cap6000 & 5795 & 3 & 64 & -2451377 & -2451377 & 43.1 & 0.000 \\
gesa2-o & 75541 & 171 & 224 & 25779856.37 & 25779856.37 & 266.1 & 0.000 \\
mas74 & 5617233 & 0 & 226 & 11801.18573 & 11801.18573 & 2884.3 & 0.000 \\
mas76 & 578986 & 0 & 212 & 40005.05414 & 40005.05414 & 276.7 & 0.000 \\
misc07 & 43418 & 71 & 380 & 2810 & 2810 & 111.8 & 0.000 \\
pk1 & 414067 & 0 & 288 & 11 & 11 & 261.4 & 0.000 \\
pp08aCUTS & 244 & 494 & 332 & 7350 & 7350 & 3.4 & 0.000 \\
qiu & 23227 & 1 & 271 & -132.8731399 & -132.8731399 & 532.9 & 0.000 \\
rout & 207437 & 68 & 266 & 1077.56 & 1077.56 & 490.1 & 0.000 \\
vpm2 & 12462 & 214 & 250 & 13.75 & 13.75 & 15.9 & 0.000 \\
ran8x32 & 32549 & 93 & 192 & 5247 & 5247 & 62.7 & 0.000 \\
ran10x26 & 78545 & 56 & 240 & 4270 & 4270 & 120.6 & 0.000 \\
ran12x21 & 162745 & 62 & 247 & 3664 & 3664 & 250.0 & 0.000 \\
ran13x13 & 101572 & 78 & 202 & 3252 & 3252 & 129.9 & 0.000 \\
mas284 & 21437 & 0 & 253 & 91405.72368 & 91405.72368 & 24.9 & 0.000 \\
prod1 & 64223 & 167 & 43 & -56 & -56 & 98.0 & 0.000 \\
bc1 & 40237 & 0 & 137 & 3.338362548 & 3.338362548 & 1234.2 & 0.000 \\
bienst1 & 9047 & 23 & 240 & 46.75 & 46.75 & 85.2 & 0.000 \\
neos2 & 408051 & 29 & 542 & 454.864697 & 454.864697 & 1871.5 & 0.000 \\
neos3 & 568518 & 42 & 137 & 30.55608862 & 422.2833322 & 3600.0 & 1281.994 \\
neos7 & 746440 & 112 & 395 & 720799.3672 & 721934 & 3600.0 & 0.157 \\
swath1 & 68506 & 59 & 191 & 379.0712957 & 379.0712957 & 599.9 & 0.000 \\
swath2 & 220028 & 80 & 213 & 385.1996929 & 385.1996929 & 2545.2 & 0.000 \\
\hline Total (24) & 9698397 & 1845 & 5792 & & & 19487.8 & 1282.151 \\
Geom. Mean & 79535.5 & & 216.2 & & & 249.3 & \\
\hline & & & & & & &
\end{tabular}

Table 30: hybrid strong/pseudocost branching $(d=5, \lambda=4)$ 


\begin{tabular}{lrrrrrrr}
\hline Example & B \& B & Cuts & StrBra & Dual Bound & Primal Bound & Time & Gap \% \\
\hline aflow30a & 164112 & 22 & 3625 & 1158 & 1158 & 364.6 & 0.000 \\
cap6000 & 4957 & 3 & 762 & -2451377 & -2451377 & 41.1 & 0.000 \\
gesa2-o & 75141 & 171 & 2231 & 25779856.37 & 25779856.37 & 275.0 & 0.000 \\
mas74 & 5163685 & 0 & 6609 & 11801.18573 & 11801.18573 & 2750.3 & 0.000 \\
mas76 & 587813 & 0 & 6307 & 40005.05414 & 40005.05414 & 274.5 & 0.000 \\
misc07 & 49822 & 71 & 3120 & 2810 & 2810 & 141.6 & 0.000 \\
pk1 & 294469 & 0 & 7008 & 11 & 11 & 154.3 & 0.000 \\
pp08aCUTS & 355 & 494 & 954 & 7350 & 7350 & 7.8 & 0.000 \\
qiu & 25180 & 1 & 6879 & -132.8731547 & -132.8731547 & 927.4 & 0.000 \\
rout & 45457 & 68 & 3004 & 1077.56 & 1077.56 & 150.0 & 0.000 \\
vpm2 & 11710 & 214 & 1948 & 13.75 & 13.75 & 18.2 & 0.000 \\
ran8x32 & 29957 & 93 & 1770 & 5247 & 5247 & 62.4 & 0.000 \\
ran10x26 & 54965 & 56 & 3038 & 4270 & 4270 & 89.0 & 0.000 \\
ran12x21 & 126153 & 62 & 5414 & 3664 & 3664 & 208.9 & 0.000 \\
ran13x13 & 86648 & 78 & 5304 & 3252 & 3252 & 132.8 & 0.000 \\
mas284 & 18700 & 0 & 4350 & 91405.72368 & 91405.72368 & 28.5 & 0.000 \\
prod1 & 72890 & 167 & 151 & -56 & -56 & 112.9 & 0.000 \\
bc1 & 42882 & 0 & 804 & 3.338362548 & 3.338362548 & 1337.0 & 0.000 \\
bienst1 & 13594 & 23 & 4150 & 46.75 & 46.75 & 121.3 & 0.000 \\
neos2 & 195730 & 29 & 1076 & 454.864697 & 454.864697 & 693.6 & 0.000 \\
neos3 & 505184 & 42 & 1264 & -318.0996496 & 448.4167142 & 3600.0 & 240.967 \\
neos7 & 546623 & 112 & 1058 & 717934 & 721934 & 3600.0 & 0.557 \\
swath1 & 64320 & 59 & 1682 & 379.0712957 & 379.0712957 & 566.0 & 0.000 \\
swath2 & 71595 & 80 & 2304 & 385.1996929 & 385.1996929 & 842.3 & 0.000 \\
\hline Total (24) & 8251942 & 1845 & 74812 & & & 16499.4 & 241.525 \\
Geom. Mean & 65966.3 & & 2284.2 & & & 229.6 & \\
\hline & & & & & & &
\end{tabular}

Table 31: hybrid strong/pseudocost branching $(d=10, \lambda=4)$ 


\begin{tabular}{lrrrrrrr}
\hline Example & B \& B & Cuts & StrBra & Dual Bound & Primal Bound & Time & Gap $\%$ \\
\hline aflow30a & 141456 & 22 & 18452 & 1158 & 1158 & 355.3 & 0.000 \\
cap6000 & 3955 & 3 & 2393 & -2451377 & -2451377 & 47.5 & 0.000 \\
gesa2-o & 66221 & 171 & 7344 & 25779856.37 & 25779856.37 & 257.1 & 0.000 \\
mas74 & 4770449 & 0 & 166155 & 11801.18573 & 11801.18573 & 2445.9 & 0.000 \\
mas76 & 577955 & 0 & 90864 & 40005.05414 & 40005.05414 & 300.4 & 0.000 \\
misc07 & 47707 & 71 & 7569 & 2810 & 2810 & 147.7 & 0.000 \\
pk1 & 309525 & 0 & 54336 & 11 & 11 & 197.4 & 0.000 \\
pp08aCUTS & 217 & 494 & 1380 & 7350 & 7350 & 9.6 & 0.000 \\
qiu & 12202 & 1 & 32215 & -132.8731475 & -132.8731475 & 1712.9 & 0.000 \\
rout & 21694 & 68 & 8874 & 1077.56 & 1077.56 & 125.1 & 0.000 \\
vpm2 & 6814 & 214 & 5182 & 13.75 & 13.75 & 18.4 & 0.000 \\
ran8x32 & 25919 & 93 & 7499 & 5247 & 5247 & 63.8 & 0.000 \\
ran10x26 & 46276 & 56 & 8639 & 4270 & 4270 & 88.0 & 0.000 \\
ran12x21 & 113911 & 62 & 26007 & 3664 & 3664 & 229.6 & 0.000 \\
ran13x13 & 78959 & 78 & 25368 & 3252 & 3252 & 149.3 & 0.000 \\
mas284 & 15250 & 0 & 17266 & 91405.72368 & 91405.72368 & 41.8 & 0.000 \\
prod1 & 73672 & 167 & 367 & -56 & -56 & 113.4 & 0.000 \\
bc1 & 35911 & 0 & 1919 & 3.338362548 & 3.338362548 & 1175.4 & 0.000 \\
bienst1 & 13775 & 23 & 17996 & 46.75 & 46.75 & 224.2 & 0.000 \\
neos2 & 188779 & 29 & 1507 & 454.864697 & 454.864697 & 755.9 & 0.000 \\
neos3 & 512536 & 42 & 1237 & -430.8470911 & 455.5620833 & 3600.0 & 205.736 \\
neos7 & 726355 & 112 & 1019 & 720052.1143 & 721934 & 3600.0 & 0.261 \\
swath1 & 65350 & 59 & 9115 & 379.0712957 & 379.0712957 & 642.1 & 0.000 \\
swath2 & 127959 & 80 & 10674 & 385.1996929 & 385.1996929 & 1554.6 & 0.000 \\
\hline Total (24) & 7982847 & 1845 & 523377 & & & 17855.3 & 205.998 \\
Geom. Mean & 57976.8 & & 8137.8 & & & 258.8 & \\
\hline & & & & & & &
\end{tabular}

Table 32: hybrid strong/pseudocost branching $(d=15, \lambda=4)$ 


\begin{tabular}{lrrrrrrr}
\hline Example & B \& B & Cuts & StrBra & Dual Bound & Primal Bound & Time & Gap \% \\
\hline aflow30a & 118518 & 22 & 46475 & 1158 & 1158 & 367.3 & 0.000 \\
cap6000 & 3803 & 3 & 3476 & -2451377 & -2451377 & 52.7 & 0.000 \\
gesa2-o & 47928 & 171 & 17595 & 25779856.37 & 25779856.37 & 233.3 & 0.000 \\
mas74 & 4883991 & 0 & 1796527 & 11744.31645 & 11893.87257 & 3646.8 & 1.273 \\
mas76 & 419280 & 0 & 404979 & 40005.05414 & 40005.05414 & 342.9 & 0.000 \\
misc07 & 42348 & 71 & 15302 & 2810 & 2810 & 157.3 & 0.000 \\
pk1 & 310978 & 0 & 169842 & 11 & 11 & 286.3 & 0.000 \\
pp08aCUTS & 254 & 494 & 1953 & 7350 & 7350 & 12.5 & 0.000 \\
qiu & 6934 & 1 & 44470 & -132.8731482 & -132.8731482 & 1799.6 & 0.000 \\
rout & 13497 & 68 & 26518 & 1077.56 & 1077.56 & 177.9 & 0.000 \\
vpm2 & 3793 & 214 & 8869 & 13.75 & 13.75 & 20.9 & 0.000 \\
ran8x32 & 19105 & 93 & 18153 & 5247 & 5247 & 75.1 & 0.000 \\
ran10x26 & 43054 & 56 & 20283 & 4270 & 4270 & 109.2 & 0.000 \\
ran12x21 & 82121 & 62 & 59947 & 3664 & 3664 & 259.5 & 0.000 \\
ran13x13 & 66732 & 78 & 74717 & 3252 & 3252 & 214.9 & 0.000 \\
mas284 & 8219 & 0 & 32171 & 91405.72368 & 91405.72368 & 50.1 & 0.000 \\
prod1 & 62571 & 167 & 1017 & -56 & -56 & 93.0 & 0.000 \\
bc1 & 33729 & 0 & 3338 & 3.338362548 & 3.338362548 & 1186.7 & 0.000 \\
bienst1 & 5677 & 23 & 28052 & 46.75 & 46.75 & 248.7 & 0.000 \\
neos2 & 427739 & 29 & 1185 & 454.864697 & 454.864697 & 1430.8 & 0.000 \\
neos3 & 611026 & 42 & 1692 & -351.7966207 & 420.0806155 & 3600.0 & 219.410 \\
neos7 & 464298 & 112 & 1768 & 721934 & 721934 & 2059.9 & 0.000 \\
swath1 & 45858 & 59 & 17054 & 379.0712957 & 379.0712957 & 532.9 & 0.000 \\
swath2 & 168921 & 80 & 29717 & 385.1996929 & 385.1996929 & 2217.6 & 0.000 \\
\hline Total (24) & 7890374 & 1845 & 2825100 & & & 19175.6 & 220.684 \\
Geom. Mean & 47958.5 & & 17780.2 & & & 293.5 & \\
\hline & & & & & &
\end{tabular}

Table 33: hybrid strong/pseudocost branching $(d=20, \lambda=4)$ 


\begin{tabular}{lrrrrrrr}
\hline Example & B \& B & Cuts & StrBra & Dual Bound & Primal Bound & Time & Gap \% \\
\hline aflow30a & 44455 & 22 & 239787 & 1158 & 1158 & 704.7 & 0.000 \\
cap6000 & 3452 & 3 & 4683 & -2451377 & -2451377 & 58.9 & 0.000 \\
gesa2-o & 15831 & 171 & 114464 & 25779856.37 & 25779856.37 & 463.7 & 0.000 \\
mas74 & 790014 & 0 & 6094848 & 11548.32371 & 11801.18573 & 3600.0 & 2.190 \\
mas76 & 125516 & 0 & 861005 & 40005.05414 & 40005.05414 & 420.2 & 0.000 \\
misc07 & 5228 & 71 & 65988 & 2810 & 2810 & 213.3 & 0.000 \\
pk1 & 128299 & 0 & 879768 & 11 & 11 & 658.1 & 0.000 \\
pp08aCUTS & 201 & 494 & 1498 & 7350 & 7350 & 10.3 & 0.000 \\
qiu & 6178 & 1 & 47170 & -132.8731482 & -132.8731482 & 1901.9 & 0.000 \\
rout & 4179 & 68 & 58916 & 1077.56 & 1077.56 & 254.7 & 0.000 \\
vpm2 & 1974 & 214 & 12712 & 13.75 & 13.75 & 25.4 & 0.000 \\
ran8x32 & 6824 & 93 & 63719 & 5247 & 5247 & 141.8 & 0.000 \\
ran10x26 & 8279 & 56 & 88240 & 4270 & 4270 & 183.1 & 0.000 \\
ran12x21 & 12185 & 62 & 141880 & 3664 & 3664 & 317.4 & 0.000 \\
ran13x13 & 16033 & 78 & 144070 & 3252 & 3252 & 233.7 & 0.000 \\
mas284 & 4564 & 0 & 32685 & 91405.72368 & 91405.72368 & 48.5 & 0.000 \\
prod1 & 14670 & 167 & 118575 & -56 & -56 & 287.1 & 0.000 \\
bc1 & 3666 & 0 & 16891 & 3.338362548 & 3.338362548 & 1108.2 & 0.000 \\
bienst1 & 5274 & 23 & 27841 & 46.75 & 46.75 & 256.8 & 0.000 \\
neos2 & 4405 & 29 & 44008 & 454.864697 & 454.864697 & 277.2 & 0.000 \\
neos3 & 13215 & 42 & 170679 & 368.842751 & 368.842751 & 1284.6 & 0.000 \\
neos7 & 55369 & 112 & 344673 & 708069.7944 & 721934 & 3600.2 & 1.958 \\
swath1 & 11268 & 59 & 80944 & 379.0712957 & 379.0712957 & 777.6 & 0.000 \\
swath2 & 44510 & 80 & 310410 & 376.4462593 & 387.6584702 & 3600.0 & 2.978 \\
\hline Total (24) & 1325589 & 1845 & 9965454 & & & 20427.2 & 7.126 \\
Geom. Mean & 11639.5 & & 86188.6 & & & 353.2 & \\
\hline & & & & & &
\end{tabular}

Table 34: hybrid strong/pseudocost branching $(d=\infty, \lambda=4)$ 


\begin{tabular}{lrrrrrrr}
\hline Example & B \& B & Cuts & StrBra & Dual Bound & Primal Bound & Time & Gap \% \\
\hline aflow30a & 217744 & 22 & 384 & 1158 & 1158 & 478.8 & 0.000 \\
cap6000 & 5795 & 3 & 64 & -2451377 & -2451377 & 41.0 & 0.000 \\
gesa2-o & 66015 & 171 & 356 & 25779856.37 & 25779856.37 & 233.6 & 0.000 \\
mas74 & 5236627 & 0 & 337 & 11801.18573 & 11801.18573 & 2964.2 & 0.000 \\
mas76 & 543507 & 0 & 325 & 40005.05414 & 40005.05414 & 255.9 & 0.000 \\
misc07 & 41476 & 71 & 449 & 2810 & 2810 & 111.0 & 0.000 \\
pk1 & 341929 & 0 & 398 & 11 & 11 & 184.4 & 0.000 \\
pp08aCUTS & 466 & 494 & 309 & 7350 & 7350 & 4.8 & 0.000 \\
qiu & 20408 & 1 & 524 & -132.8731473 & -132.8731473 & 501.9 & 0.000 \\
rout & 95870 & 68 & 462 & 1077.56 & 1077.56 & 264.0 & 0.000 \\
vpm2 & 19796 & 214 & 389 & 13.75 & 13.75 & 26.0 & 0.000 \\
ran8x32 & 38242 & 93 & 366 & 5247 & 5247 & 76.3 & 0.000 \\
ran10x26 & 59476 & 56 & 331 & 4270 & 4270 & 95.3 & 0.000 \\
ran12x21 & 140140 & 62 & 386 & 3664 & 3664 & 238.5 & 0.000 \\
ran13x13 & 93526 & 78 & 381 & 3252 & 3252 & 137.3 & 0.000 \\
mas284 & 22188 & 0 & 398 & 91405.72368 & 91405.72368 & 26.4 & 0.000 \\
prod1 & 66116 & 163 & 88 & -56 & -56 & 101.7 & 0.000 \\
bc1 & 40237 & 0 & 137 & 3.338362548 & 3.338362548 & 1252.8 & 0.000 \\
bienst1 & 8277 & 23 & 377 & 46.75 & 46.75 & 80.4 & 0.000 \\
neos2 & 529779 & 29 & 152 & 454.864697 & 454.864697 & 2125.7 & 0.000 \\
neos3 & 463388 & 42 & 113 & -254.4223987 & 457.6576489 & 3600.0 & 279.881 \\
neos7 & 342560 & 112 & 84 & 721934 & 721934 & 1657.4 & 0.000 \\
swath1 & 62909 & 59 & 274 & 379.0712957 & 379.0712957 & 559.1 & 0.000 \\
swath2 & 196847 & 80 & 313 & 385.1996929 & 385.1996929 & 2372.9 & 0.000 \\
\hline Total (24) & 8653318 & 1841 & 7397 & & & 17389.5 & 279.881 \\
Geom. Mean & 74730.8 & & 268.6 & & & 239.6 & \\
\hline & & & & & & &
\end{tabular}

Table 35: hybrid strong/pseudocost branching $(d=5, \lambda=8)$ 


\begin{tabular}{lrrrrrrr}
\hline Example & B \& B & Cuts & StrBra & Dual Bound & Primal Bound & Time & Gap $\%$ \\
\hline aflow30a & 210127 & 22 & 5604 & 1158 & 1158 & 457.6 & 0.000 \\
cap6000 & 4957 & 3 & 762 & -2451377 & -2451377 & 40.7 & 0.000 \\
gesa2-o & 74673 & 171 & 3373 & 25779856.37 & 25779856.37 & 272.5 & 0.000 \\
mas74 & 4886438 & 0 & 10531 & 11801.18573 & 11801.18573 & 2902.2 & 0.000 \\
mas76 & 666398 & 0 & 9080 & 40005.05414 & 40005.05414 & 315.3 & 0.000 \\
misc07 & 50893 & 71 & 2943 & 2810 & 2810 & 143.2 & 0.000 \\
pk1 & 298771 & 0 & 9334 & 11 & 11 & 157.2 & 0.000 \\
pp08aCUTS & 318 & 494 & 1531 & 7350 & 7350 & 10.3 & 0.000 \\
qiu & 23974 & 1 & 11732 & -132.8731746 & -132.8731746 & 1213.9 & 0.000 \\
rout & 39212 & 68 & 5554 & 1077.56 & 1077.56 & 165.2 & 0.000 \\
vpm2 & 10826 & 214 & 2971 & 13.75 & 13.75 & 19.1 & 0.000 \\
ran8x32 & 30526 & 93 & 2765 & 5247 & 5247 & 59.3 & 0.000 \\
ran10x26 & 56448 & 56 & 3771 & 4270 & 4270 & 100.3 & 0.000 \\
ran12x21 & 135165 & 62 & 6588 & 3664 & 3664 & 222.2 & 0.000 \\
ran13x13 & 86566 & 78 & 7920 & 3252 & 3252 & 134.4 & 0.000 \\
mas284 & 17799 & 0 & 5010 & 91405.72368 & 91405.72368 & 27.8 & 0.000 \\
prod1 & 69992 & 163 & 335 & -56 & -56 & 101.1 & 0.000 \\
bc1 & 40212 & 0 & 787 & 3.338362548 & 3.338362548 & 1282.2 & 0.000 \\
bienst1 & 13602 & 23 & 4380 & 46.75 & 46.75 & 138.4 & 0.000 \\
neos2 & 244292 & 29 & 267 & 454.864697 & 454.864697 & 883.6 & 0.000 \\
neos3 & 536938 & 42 & 270 & -98.44534014 & 463.9424776 & 3600.0 & 571.269 \\
neos7 & 598209 & 112 & 171 & 720799.3672 & 721934 & 3600.0 & 0.157 \\
swath1 & 73512 & 59 & 3235 & 379.0712957 & 379.0712957 & 668.6 & 0.000 \\
swath2 & 162695 & 80 & 3168 & 385.1996929 & 385.1996929 & 1847.6 & 0.000 \\
\hline Total (24) & 8332543 & 1841 & 102082 & & & 18362.5 & 571.427 \\
Geom. Mean & 69489.0 & & 2523.8 & & & 253.9 & \\
\hline & & & & & & &
\end{tabular}

Table 36: hybrid strong/pseudocost branching $(d=10, \lambda=8)$ 


\begin{tabular}{lrrrrrrr}
\hline Example & B \& B & Cuts & StrBra & Dual Bound & Primal Bound & Time & Gap \% \\
\hline aflow30a & 160260 & 22 & 24185 & 1158 & 1158 & 413.8 & 0.000 \\
cap6000 & 3955 & 3 & 2393 & -2451377 & -2451377 & 46.0 & 0.000 \\
gesa2-o & 42668 & 171 & 12242 & 25779856.37 & 25779856.37 & 203.3 & 0.000 \\
mas74 & 4032872 & 0 & 228138 & 11801.18573 & 11801.18573 & 2096.5 & 0.000 \\
mas76 & 673256 & 0 & 115749 & 40005.05414 & 40005.05414 & 354.9 & 0.000 \\
misc07 & 48670 & 71 & 8643 & 2810 & 2810 & 160.0 & 0.000 \\
pk1 & 395401 & 0 & 102137 & 11 & 11 & 303.2 & 0.000 \\
pp08aCUTS & 237 & 494 & 1938 & 7350 & 7350 & 12.8 & 0.000 \\
qiu & 11253 & 1 & 75265 & -156.7635313 & -132.8731682 & 3600.0 & 15.240 \\
rout & 20435 & 68 & 15955 & 1077.56 & 1077.56 & 171.1 & 0.000 \\
vpm2 & 6964 & 214 & 7652 & 13.75 & 13.75 & 25.9 & 0.000 \\
ran8x32 & 24083 & 93 & 8584 & 5247 & 5247 & 60.9 & 0.000 \\
ran10x26 & 48889 & 56 & 14049 & 4270 & 4270 & 116.1 & 0.000 \\
ran12x21 & 108774 & 62 & 29130 & 3664 & 3664 & 232.7 & 0.000 \\
ran13x13 & 77781 & 78 & 33649 & 3252 & 3252 & 166.4 & 0.000 \\
mas284 & 13657 & 0 & 20339 & 91405.72368 & 91405.72368 & 44.5 & 0.000 \\
prod1 & 67428 & 163 & 949 & -56 & -56 & 105.1 & 0.000 \\
bc1 & 35537 & 0 & 1936 & 3.338362548 & 3.338362548 & 1171.6 & 0.000 \\
bienst1 & 16257 & 23 & 17816 & 46.75 & 46.75 & 224.1 & 0.000 \\
neos2 & 247754 & 29 & 637 & 454.864697 & 454.864697 & 897.6 & 0.000 \\
neos3 & 579422 & 42 & 449 & -8.446167301 & 464.5073112 & 3600.0 & 5599.622 \\
neos7 & 532834 & 112 & 740 & 721934 & 721934 & 2741.9 & 0.000 \\
swath1 & 72628 & 59 & 14180 & 379.0712957 & 379.0712957 & 803.8 & 0.000 \\
swath2 & 235670 & 80 & 13822 & 385.1996929 & 385.1996929 & 2927.6 & 0.000 \\
\hline Total (24) & 7456685 & 1841 & 750577 & & & 20479.8 & 5614.862 \\
Geom. Mean & 59398.8 & & 9983.1 & & & 295.7 & \\
\hline
\end{tabular}

Table 37: hybrid strong/pseudocost branching $(d=15, \lambda=8)$ 


\begin{tabular}{lrrrrrrr}
\hline Example & B \& B & Cuts & StrBra & Dual Bound & Primal Bound & Time & Gap \% \\
\hline aflow30a & 156348 & 22 & 63565 & 1158 & 1158 & 505.6 & 0.000 \\
cap6000 & 3803 & 3 & 3476 & -2451377 & -2451377 & 52.4 & 0.000 \\
gesa2-o & 45713 & 171 & 23564 & 25779856.37 & 25779856.37 & 260.7 & 0.000 \\
mas74 & 4549153 & 0 & 2165284 & 11783.43461 & 11801.18573 & 3600.0 & 0.151 \\
mas76 & 586552 & 0 & 591079 & 40005.05414 & 40005.05414 & 515.8 & 0.000 \\
misc07 & 42015 & 71 & 18401 & 2810 & 2810 & 179.0 & 0.000 \\
pk1 & 301968 & 0 & 352125 & 11 & 11 & 413.7 & 0.000 \\
pp08aCUTS & 148 & 494 & 1530 & 7350 & 7350 & 10.1 & 0.000 \\
qiu & 6371 & 1 & 84603 & -195.1941608 & -132.8731568 & 3600.1 & 31.928 \\
rout & 23134 & 68 & 46443 & 1077.56 & 1077.56 & 331.2 & 0.000 \\
vpm2 & 3709 & 214 & 13554 & 13.75 & 13.75 & 31.5 & 0.000 \\
ran8x32 & 19377 & 93 & 16810 & 5247 & 5247 & 73.7 & 0.000 \\
ran10x26 & 45080 & 56 & 28137 & 4270 & 4270 & 136.2 & 0.000 \\
ran12x21 & 85741 & 62 & 63641 & 3664 & 3664 & 280.9 & 0.000 \\
ran13x13 & 66793 & 78 & 75150 & 3252 & 3252 & 217.1 & 0.000 \\
mas284 & 9416 & 0 & 43047 & 91405.72368 & 91405.72368 & 67.9 & 0.000 \\
prod1 & 61573 & 163 & 3224 & -56 & -56 & 102.7 & 0.000 \\
bc1 & 35167 & 0 & 3373 & 3.338362548 & 3.338362548 & 1228.4 & 0.000 \\
bienst1 & 5907 & 23 & 30012 & 46.75 & 46.75 & 277.4 & 0.000 \\
neos2 & 215631 & 29 & 608 & 454.864697 & 454.864697 & 695.8 & 0.000 \\
neos3 & 549304 & 42 & 515 & -80.06097644 & 484.0603447 & 3600.0 & 704.615 \\
neos7 & 444334 & 112 & 1217 & 721934 & 721934 & 2329.4 & 0.000 \\
swath1 & 57789 & 59 & 26643 & 379.0712957 & 379.0712957 & 735.9 & 0.000 \\
swath2 & 236393 & 80 & 39576 & 385.1996929 & 385.1996929 & 3143.4 & 0.000 \\
\hline Total (24) & 7551419 & 1841 & 3695577 & & & 22388.8 & 736.693 \\
Geom. Mean & 48736.5 & & 20837.9 & & & 343.1 & \\
\hline & & & & & &
\end{tabular}

Table 38: hybrid strong/pseudocost branching $(d=20, \lambda=8)$ 


\begin{tabular}{lrrrrrrr}
\hline Example & B \& B & Cuts & StrBra & Dual Bound & Primal Bound & Time & Gap \% \\
\hline aflow30a & 55478 & 22 & 495934 & 1158 & 1158 & 1522.8 & 0.000 \\
cap6000 & 3452 & 3 & 4683 & -2451377 & -2451377 & 59.5 & 0.000 \\
gesa2-o & 21988 & 171 & 235608 & 25779856.37 & 25779856.37 & 926.6 & 0.000 \\
mas74 & 645364 & 0 & 6617267 & 11513.08574 & 12292.91697 & 3600.0 & 6.773 \\
mas76 & 188882 & 0 & 1551253 & 40005.05414 & 40005.05414 & 713.9 & 0.000 \\
misc07 & 5394 & 71 & 84665 & 2810 & 2810 & 267.4 & 0.000 \\
pk1 & 143206 & 0 & 1256800 & 11 & 11 & 885.0 & 0.000 \\
pp08aCUTS & 148 & 494 & 1530 & 7350 & 7350 & 9.9 & 0.000 \\
qiu & 6416 & 1 & 87289 & -193.5047713 & -132.8731475 & 3600.4 & 31.333 \\
rout & 11305 & 68 & 210641 & 1077.56 & 1077.56 & 785.8 & 0.000 \\
vpm2 & 1812 & 214 & 15435 & 13.75 & 13.75 & 30.9 & 0.000 \\
ran8x32 & 4249 & 93 & 58732 & 5247 & 5247 & 133.0 & 0.000 \\
ran10x26 & 11112 & 56 & 144009 & 4270 & 4270 & 294.2 & 0.000 \\
ran12x21 & 18577 & 62 & 269767 & 3664 & 3664 & 529.3 & 0.000 \\
ran13x13 & 20516 & 78 & 234413 & 3252 & 3252 & 355.0 & 0.000 \\
mas284 & 5580 & 0 & 47686 & 91405.72368 & 91405.72368 & 71.0 & 0.000 \\
prod1 & 15609 & 163 & 152860 & -56 & -56 & 359.3 & 0.000 \\
bc1 & 3603 & 0 & 16810 & 3.338362548 & 3.338362548 & 1071.5 & 0.000 \\
bienst1 & 5340 & 23 & 30331 & 46.75 & 46.75 & 270.3 & 0.000 \\
neos2 & 2685 & 29 & 37362 & 454.864697 & 454.864697 & 216.9 & 0.000 \\
neos3 & 15306 & 42 & 223816 & 368.842751 & 368.842751 & 1550.2 & 0.000 \\
neos7 & 55799 & 112 & 417934 & 709654.4626 & 721934 & 3600.1 & 1.730 \\
swath1 & 16471 & 59 & 129431 & 379.0712957 & 379.0712957 & 1166.1 & 0.000 \\
swath2 & 36277 & 80 & 327248 & 373.4967249 & 389.5079992 & 3600.3 & 4.287 \\
\hline Total (24) & 1294569 & 1841 & 12651504 & & & 25619.4 & 44.124 \\
Geom. Mean & 12714.7 & & 119799.1 & & & 468.0 & \\
\hline & & & & & & &
\end{tabular}

Table 39: hybrid strong/pseudocost branching $(d=\infty, \lambda=8)$ 


\begin{tabular}{lrrrrrrr}
\hline Example & B \& B & Cuts & StrBra & Dual Bound & Primal Bound & Time & Gap \% \\
\hline aflow30a & 152417 & 22 & 1003 & 1158 & 1158 & 368.8 & 0.000 \\
cap6000 & 5795 & 3 & 64 & -2451377 & -2451377 & 42.2 & 0.000 \\
gesa2-o & 48481 & 171 & 1484 & 25779856.37 & 25779856.37 & 174.2 & 0.000 \\
mas74 & 4817986 & 0 & 372 & 11801.18573 & 11801.18573 & 2526.9 & 0.000 \\
mas76 & 487621 & 0 & 341 & 40005.05414 & 40005.05414 & 222.2 & 0.000 \\
misc07 & 52755 & 71 & 585 & 2810 & 2810 & 138.6 & 0.000 \\
pk1 & 376201 & 0 & 442 & 11 & 11 & 202.8 & 0.000 \\
pp08aCUTS & 446 & 494 & 363 & 7350 & 7350 & 4.7 & 0.000 \\
qiu & 45147 & 1 & 1054 & -132.8731782 & -132.8731782 & 875.3 & 0.000 \\
rout & 106110 & 68 & 1039 & 1077.56 & 1077.56 & 300.6 & 0.000 \\
vpm2 & 12637 & 214 & 718 & 13.75 & 13.75 & 18.6 & 0.000 \\
ran8x32 & 21126 & 93 & 754 & 5247 & 5247 & 41.0 & 0.000 \\
ran10x26 & 65019 & 56 & 956 & 4270 & 4270 & 110.6 & 0.000 \\
ran12x21 & 138391 & 62 & 1159 & 3664 & 3664 & 221.4 & 0.000 \\
ran13x13 & 109899 & 78 & 792 & 3252 & 3252 & 165.5 & 0.000 \\
mas284 & 18944 & 0 & 544 & 91405.72368 & 91405.72368 & 22.1 & 0.000 \\
prod1 & 67884 & 156 & 323 & -56 & -56 & 103.4 & 0.000 \\
bc1 & 40237 & 0 & 137 & 3.338362548 & 3.338362548 & 1250.6 & 0.000 \\
bienst1 & 7207 & 23 & 500 & 46.75 & 46.75 & 67.2 & 0.000 \\
neos2 & 666315 & 29 & 202 & 454.864697 & 454.864697 & 2702.4 & 0.000 \\
neos3 & 441612 & 42 & 228 & -589.5108992 & 511.5284678 & 3600.0 & 186.772 \\
neos7 & 682983 & 112 & 910 & 720460.9034 & 721934 & 3600.0 & 0.204 \\
swath1 & 51424 & 59 & 316 & 379.0712957 & 379.0712957 & 438.0 & 0.000 \\
swath2 & 81655 & 80 & 389 & 385.1996929 & 385.1996929 & 919.7 & 0.000 \\
\hline Total (24) & 8498292 & 1834 & 14675 & & & 18116.9 & 186.976 \\
Geom. Mean & 71817.4 & & 489.9 & & & 229.3 & \\
\hline & & & & & &
\end{tabular}

Table 40: hybrid strong/pseudocost branching $(d=5, \lambda=\infty)$ 


\begin{tabular}{lrrrrrrr}
\hline Example & B \& B & Cuts & StrBra & Dual Bound & Primal Bound & Time & Gap \% \\
\hline aflow30a & 147810 & 22 & 8409 & 1158 & 1158 & 383.9 & 0.000 \\
cap6000 & 4957 & 3 & 762 & -2451377 & -2451377 & 40.9 & 0.000 \\
gesa2-o & 62134 & 171 & 13652 & 25779856.37 & 25779856.37 & 260.1 & 0.000 \\
mas74 & 5815301 & 0 & 12226 & 11801.18573 & 11801.18573 & 3556.4 & 0.000 \\
mas76 & 484042 & 0 & 9273 & 40005.05414 & 40005.05414 & 202.5 & 0.000 \\
misc07 & 49998 & 71 & 3582 & 2810 & 2810 & 143.0 & 0.000 \\
pk1 & 376305 & 0 & 11658 & 11 & 11 & 206.8 & 0.000 \\
pp08aCUTS & 280 & 494 & 1599 & 7350 & 7350 & 10.8 & 0.000 \\
qiu & 31784 & 1 & 25993 & -132.8731384 & -132.8731384 & 2177.4 & 0.000 \\
rout & 48147 & 68 & 7291 & 1077.56 & 1077.56 & 202.8 & 0.000 \\
vpm2 & 11167 & 214 & 4659 & 13.75 & 13.75 & 25.6 & 0.000 \\
ran8x32 & 20044 & 93 & 3327 & 5247 & 5247 & 46.9 & 0.000 \\
ran10x26 & 74615 & 56 & 7234 & 4270 & 4270 & 135.1 & 0.000 \\
ran12x21 & 124672 & 62 & 10512 & 3664 & 3664 & 234.0 & 0.000 \\
ran13x13 & 103787 & 78 & 11794 & 3252 & 3252 & 172.8 & 0.000 \\
mas284 & 19852 & 0 & 6421 & 91405.72368 & 91405.72368 & 35.8 & 0.000 \\
prod1 & 65005 & 156 & 785 & -56 & -56 & 100.5 & 0.000 \\
bc1 & 40212 & 0 & 787 & 3.338362548 & 3.338362548 & 1292.7 & 0.000 \\
bienst1 & 13421 & 23 & 4634 & 46.75 & 46.75 & 135.0 & 0.000 \\
neos2 & 482124 & 29 & 398 & 454.864697 & 454.864697 & 1783.5 & 0.000 \\
neos3 & 496138 & 42 & 439 & -265.6892095 & 471.2518713 & 3600.0 & 277.370 \\
neos7 & 576776 & 112 & 1693 & 719949.0129 & 721934 & 3600.0 & 0.276 \\
swath1 & 75401 & 59 & 3623 & 379.0712957 & 379.0712957 & 683.7 & 0.000 \\
swath2 & 123664 & 80 & 3707 & 385.1996929 & 385.1996929 & 1442.0 & 0.000 \\
\hline Total (24) & 9247636 & 1834 & 154458 & & & 20472.1 & 277.645 \\
Geom. Mean & 70125.8 & & 3870.1 & & & 276.2 & \\
\hline & & & & & &
\end{tabular}

Table 41: hybrid strong/pseudocost branching $(d=10, \lambda=\infty)$ 


\begin{tabular}{lrrrrrrr}
\hline Example & B \& B & Cuts & StrBra & Dual Bound & Primal Bound & Time & Gap $\%$ \\
\hline aflow30a & 147861 & 22 & 31388 & 1158 & 1158 & 452.3 & 0.000 \\
cap6000 & 3955 & 3 & 2393 & -2451377 & -2451377 & 45.4 & 0.000 \\
gesa2-o & 61403 & 171 & 37626 & 25779856.37 & 25779856.37 & 350.7 & 0.000 \\
mas74 & 3293309 & 0 & 253958 & 11801.18573 & 11801.18573 & 1647.5 & 0.000 \\
mas76 & 685295 & 0 & 120302 & 40005.05414 & 40005.05414 & 355.8 & 0.000 \\
misc07 & 49496 & 71 & 9884 & 2810 & 2810 & 164.4 & 0.000 \\
pk1 & 386692 & 0 & 119283 & 11 & 11 & 295.6 & 0.000 \\
pp08aCUTS & 185 & 494 & 1568 & 7350 & 7350 & 10.1 & 0.000 \\
qiu & 3922 & 1 & 72833 & -297.9264066 & -119.2175182 & 3601.6 & 59.984 \\
rout & 31930 & 68 & 27218 & 1077.56 & 1077.56 & 286.8 & 0.000 \\
vpm2 & 7791 & 214 & 10279 & 13.75 & 13.75 & 32.2 & 0.000 \\
ran8x32 & 20310 & 93 & 10437 & 5247 & 5247 & 62.9 & 0.000 \\
ran10x26 & 69082 & 56 & 21054 & 4270 & 4270 & 154.7 & 0.000 \\
ran12x21 & 111742 & 62 & 41826 & 3664 & 3664 & 279.1 & 0.000 \\
ran13x13 & 84200 & 78 & 47311 & 3252 & 3252 & 200.8 & 0.000 \\
mas284 & 14532 & 0 & 22922 & 91405.72368 & 91405.72368 & 51.9 & 0.000 \\
prod1 & 63355 & 156 & 2176 & -56 & -56 & 104.1 & 0.000 \\
bc1 & 35537 & 0 & 1936 & 3.338362548 & 3.338362548 & 1176.5 & 0.000 \\
bienst1 & 15376 & 23 & 20999 & 46.75 & 46.75 & 260.2 & 0.000 \\
neos2 & 217194 & 29 & 569 & 454.864697 & 454.864697 & 680.0 & 0.000 \\
neos3 & 508961 & 42 & 647 & -144.8889495 & 484.9386703 & 3600.0 & 434.697 \\
neos7 & 648644 & 112 & 2731 & 720322.1301 & 721934 & 3600.0 & 0.224 \\
swath1 & 74370 & 59 & 15123 & 379.0712957 & 379.0712957 & 788.5 & 0.000 \\
swath2 & 135298 & 80 & 15724 & 385.1996929 & 385.1996929 & 1706.3 & 0.000 \\
\hline Total (24) & 6670440 & 1834 & 890187 & & & 19907.4 & 494.905 \\
Geom. Mean & 56926.6 & & 13127.2 & & & 312.2 & \\
\hline & & & & & &
\end{tabular}

Table 42: hybrid strong/pseudocost branching $(d=15, \lambda=\infty)$ 


\begin{tabular}{lrrrrrrr}
\hline Example & B \& B & Cuts & StrBra & Dual Bound & Primal Bound & Time & Gap \% \\
\hline aflow30a & 108726 & 22 & 79374 & 1158 & 1158 & 496.4 & 0.000 \\
cap6000 & 3803 & 3 & 3476 & -2451377 & -2451377 & 52.2 & 0.000 \\
gesa2-o & 58602 & 171 & 63948 & 25779856.37 & 25779856.37 & 431.3 & 0.000 \\
mas74 & 4468326 & 0 & 2095085 & 11801.18573 & 11801.18573 & 3260.7 & 0.000 \\
mas76 & 735551 & 0 & 709846 & 40005.05414 & 40005.05414 & 639.8 & 0.000 \\
misc07 & 42486 & 71 & 19377 & 2810 & 2810 & 173.9 & 0.000 \\
pk1 & 331170 & 0 & 308821 & 11 & 11 & 388.7 & 0.000 \\
pp08aCUTS & 219 & 494 & 2173 & 7350 & 7350 & 13.5 & 0.000 \\
qiu & 3171 & 1 & 74723 & -314.9907564 & -119.2175182 & 3600.0 & 62.152 \\
rout & 29159 & 68 & 64331 & 1077.56 & 1077.56 & 434.4 & 0.000 \\
vpm2 & 4539 & 214 & 14127 & 13.75 & 13.75 & 34.7 & 0.000 \\
ran8x32 & 14316 & 93 & 19612 & 5247 & 5247 & 70.5 & 0.000 \\
ran10x26 & 57480 & 56 & 45191 & 4270 & 4270 & 194.7 & 0.000 \\
ran12x21 & 84428 & 62 & 92214 & 3664 & 3664 & 345.6 & 0.000 \\
ran13x13 & 78723 & 78 & 93836 & 3252 & 3252 & 269.7 & 0.000 \\
mas284 & 6489 & 0 & 34688 & 91405.72368 & 91405.72368 & 56.6 & 0.000 \\
prod1 & 66236 & 156 & 6237 & -56 & -56 & 118.5 & 0.000 \\
bc1 & 35167 & 0 & 3373 & 3.338362548 & 3.338362548 & 1200.5 & 0.000 \\
bienst1 & 5810 & 23 & 30335 & 46.75 & 46.75 & 283.1 & 0.000 \\
neos2 & 153173 & 29 & 747 & 454.864697 & 454.864697 & 535.0 & 0.000 \\
neos3 & 566287 & 42 & 896 & 27.06697074 & 410.2985392 & 3600.0 & 1415.864 \\
neos7 & 498056 & 112 & 4205 & 712542.8525 & 721934 & 3600.0 & 1.318 \\
swath1 & 65320 & 59 & 29483 & 379.0712957 & 379.0712957 & 801.1 & 0.000 \\
swath2 & 210403 & 80 & 46418 & 385.1996929 & 385.1996929 & 2937.6 & 0.000 \\
\hline Total (24) & 7627640 & 1834 & 3842516 & & & 23538.5 & 1479.334 \\
Geom. Mean & 48547.0 & & 26557.4 & & & 373.6 & \\
\hline
\end{tabular}

Table 43: hybrid strong/pseudocost branching $(d=20, \lambda=\infty)$ 


\begin{tabular}{lrrrrrrr}
\hline Example & B \& B & Cuts & StrBra & Dual Bound & Primal Bound & Time & Gap \% \\
\hline aflow30a & 34855 & 22 & 602929 & 1158 & 1158 & 1669.9 & 0.000 \\
cap6000 & 3452 & 3 & 4683 & -2451377 & -2451377 & 61.7 & 0.000 \\
gesa2-o & 17294 & 171 & 335723 & 25779856.37 & 25779856.37 & 1161.4 & 0.000 \\
mas74 & 615493 & 0 & 6812753 & 11513.4239 & 11801.18573 & 3600.0 & 2.499 \\
mas76 & 164161 & 0 & 1406438 & 40005.05414 & 40005.05414 & 670.3 & 0.000 \\
misc07 & 4861 & 71 & 89217 & 2810 & 2810 & 286.8 & 0.000 \\
pk1 & 113940 & 0 & 1034246 & 11 & 11 & 716.8 & 0.000 \\
pp08aCUTS & 199 & 494 & 2144 & 7350 & 7350 & 13.1 & 0.000 \\
qiu & 3152 & 1 & 75517 & -314.0791134 & -119.2175182 & 3600.1 & 62.042 \\
rout & 7512 & 68 & 190851 & 1077.56 & 1077.56 & 801.9 & 0.000 \\
vpm2 & 2502 & 214 & 27728 & 13.75 & 13.75 & 55.3 & 0.000 \\
ran8x32 & 3674 & 93 & 61342 & 5247 & 5247 & 135.4 & 0.000 \\
ran10x26 & 12080 & 56 & 207370 & 4270 & 4270 & 408.6 & 0.000 \\
ran12x21 & 19194 & 62 & 362574 & 3664 & 3664 & 718.5 & 0.000 \\
ran13x13 & 12048 & 78 & 161604 & 3252 & 3252 & 248.2 & 0.000 \\
mas284 & 4710 & 0 & 43870 & 91405.72368 & 91405.72368 & 66.3 & 0.000 \\
prod1 & 15895 & 156 & 172732 & -56 & -56 & 401.5 & 0.000 \\
bc1 & 3603 & 0 & 16810 & 3.338362548 & 3.338362548 & 1073.0 & 0.000 \\
bienst1 & 5548 & 23 & 30317 & 46.75 & 46.75 & 278.2 & 0.000 \\
neos2 & 2683 & 29 & 45243 & 454.864697 & 454.864697 & 276.4 & 0.000 \\
neos3 & 14641 & 42 & 251155 & 368.842751 & 368.842751 & 1732.2 & 0.000 \\
neos7 & 53276 & 112 & 423037 & 709085.0815 & 721934 & 3600.0 & 1.812 \\
swath1 & 13465 & 59 & 110397 & 379.0712957 & 379.0712957 & 1000.8 & 0.000 \\
swath2 & 35584 & 80 & 324684 & 375.8885115 & 388.970984 & 3600.1 & 3.480 \\
\hline Total (24) & 1163822 & 1834 & 12793364 & & & 26176.4 & 69.834 \\
Geom. Mean & 11355.7 & & 127737.1 & & 500.3 & \\
\hline & & & & & & &
\end{tabular}

Table 44: hybrid strong/pseudocost branching $(d=\infty, \lambda=\infty)$ 